\title{
Assessment of Wake-Tail Interference Effects on the
}

\section{Dynamics of Flexible Aircraft}

\author{
Joseba Murua ${ }^{1}$ and Rafael Palacios ${ }^{2}$ and J. Michael R. Graham ${ }^{3}$ \\ Imperial College, London SW7 2AZ, United Kingdom
}

This work investigates the effect of aerodynamic interference in the coupled nonlinear aeroelasticity and flight mechanics of flexible lightweight aircraft at low speeds. For that purpose, a geometrically-exact composite beam formulation is used to model the vehicle flexible-body dynamics, by means of an intuitive and easily linearizable representation based on the displacement and Cartesian rotation vectors. The aerodynamics are modeled using the unsteady vortex-lattice method, which captures the instantaneous shape of the lifting surfaces and the free inviscid wake, including large deformations and interference effects. This results in a framework for Simulation of High Aspect Ratio Planes that provides a medium-fidelity representation of flexibleaircraft dynamics with a modest computational cost. Previous independent studies on the structural-dynamics and aerodynamics modules are complemented here with the integrated simulation methodology, including vehicle trim, and linear and nonlinear time-domain solutions. A numerical investigation is next presented on a simple wingfuselage-tail configuration, assessing the interference effects between wing-wake and horizontal tail, and the downwash due to the proximity of the wake is shown to play a significant role in the longitudinal dynamics of the vehicle. Finally, a brief discussion of direct wake-tail encounters is included to show the limitations of the approach.

\footnotetext{
${ }^{1}$ Graduate student, Department of Aeronautics, 352 Roderic Hill Building, AIAA Student Member.

2 Lecturer, Department of Aeronautics, 355 Roderic Hill Building, AIAA Member (Contact author. e-mail: rpalacio@imperial.ac.uk)

${ }^{3}$ Professor, Department of Aeronautics, 362 Roderic Hill Building.
} 


\section{Nomenclature}

$C=$ global tangent damping matrix

$C^{B a}=$ coordinate transformation matrix, from $a$ to $B$

$K=$ global tangent stiffness matrix

$m \quad=$ mass per unit length

$M=$ global tangent mass matrix

$\mathcal{M}=$ cross-sectional mass matrix

$Q=$ global vector of generalized forces

$\vec{r} \quad=$ position vector of the body-fixed frame, $a$, from the inertial one

$\vec{R} \quad=$ local position vector along the beam reference line

$s \quad=$ arc length along reference line of the beam elements

$\mathcal{S}=$ cross-sectional stiffness matrix

$t \quad=$ physical time

$T=$ tangential operator

$\vec{v} \quad=$ inertial translational velocity of the body-fixed frame, $a$

$\vec{V} \quad=$ inertial translation velocity at a beam location

$\boldsymbol{w}=$ vector of non-vortical velocities at all collocation points

$\boldsymbol{X}=$ coordinates of the aerodynamic lattice

$\alpha \quad=$ angle of incidence

$\delta \quad=$ elevator deflection

$\boldsymbol{\eta}=$ vector of displacements and rotations at all finite-element nodes

$\gamma \quad=$ beam local force strain

$\boldsymbol{\Gamma}=$ vector of circulation strengths in vortex rings

$\zeta=$ quaternions of the orientation of the body-fixed frame, $a$

$\kappa=$ beam local moment strain

$\vec{\xi}=$ relative position vector within a beam section/airfoil

$\Psi=$ Cartesian rotation vector at a given node

$\vec{\omega} \quad=$ inertial angular velocity of the body-fixed frame, $a$

$\vec{\Omega} \quad=$ inertial angular velocity at a beam location 


\section{Subscripts}

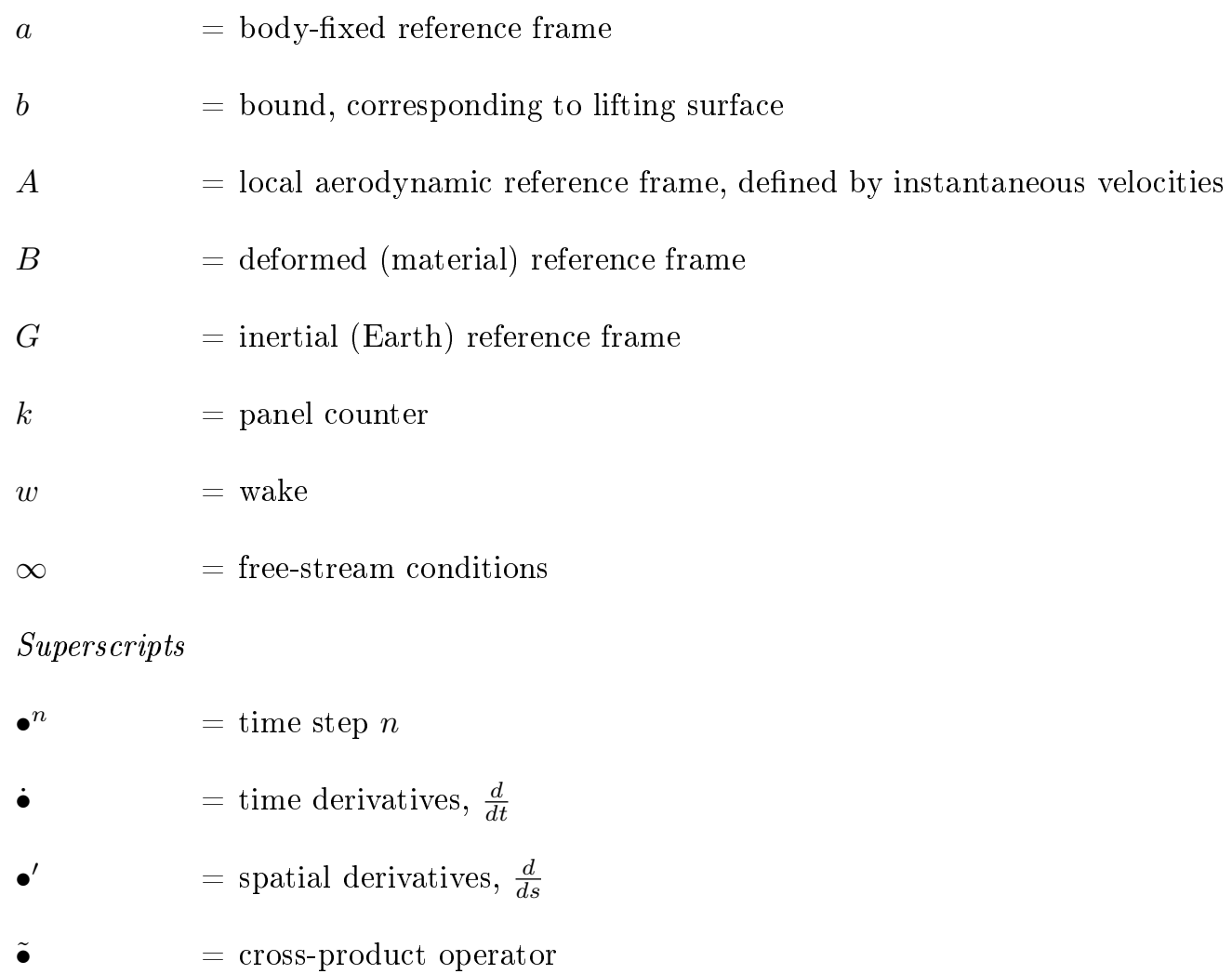

\section{Introduction}

Solar- and hydrogen-powered aircraft for commercially-viable very-long-endurance unmanned flight are defining a new frontier in the history of aviation, with several prototypes currently at different stages of development. Due to the exceptionally demanding efficiency requirements, these vehicles are built on an extremely light structure with large aspect ratio wings. This brings a number of issues that need to be considered in the design process: the possibility of large (geometricallynonlinear) structural deformations, coupling between aeroelastic and flight dynamics responses, low resilience to atmospheric turbulence and gusts, controllability issues, etc. New multidisciplinary analysis frameworks are thus needed for the aeroservoelastic design of these Very Flexible Aircraft (VFA) and a substantial research effort has been carried out towards this goal in recent years [19]. In most cases, the characteristic slenderness of all primary flexible structures of High-Altitude Long-Endurance (HALE) vehicles justifies descriptions of the low-frequency nonlinear structuraldynamics through beam models, and of the unsteady aerodynamics by means of 2-D strip theory. 
Three-dimensional aerodynamic effects may be important however in the interference between lifting surfaces and wakes (typically between the wake shed by the main wing and the tail) or in the wingtip effects. The latter is typically considered through corrections on the lift curve, but those are only valid in small ranges of reduced frequencies [10].

The primary structures of a particular VFA configuration (wing, fuselage, tail) are thus modeled as composite beams. They undergo three-dimensional displacements and rotations, with crosssectional properties calculated along the span. Typically those displacements and rotations are the primary variables in the numerical solution of the structural problem [11, 12], which is solved under the assumption of small strain. In some recent works, however, the strain of the beam elements $[7,9]$ or the local velocities and strains (the intrinsic description) $[13,14]$ have been taken as independent degrees of freedom, which can provide some numerical advantages on aircraft-type geometries. A comparative study on these different structural models for flexible-aircraft dynamics has been presented in a previous work by the authors [10].

A key driving factor in the design of large, lightweight VFA is their response to gusts and atmospheric turbulence during climb and descend operations, as illustrated by the Helios mishap [15]. HALE vehicles are likely to satisfy potential-flow assumptions (low-speed flight and attached flow) during these mission segments, hence rendering panel methods excellent candidates for the description of the aerodynamics: without incurring in excessive computational costs, they incorporate 3-D effects, interference and wake modeling. Note, however, that panel methods are not adequate at very high altitudes due to dominance of viscous effects or breakdown of continuum hypothesis.

Among potential-flow solvers, free-wake methods become necessary for complex wing kinematics, in particular, for large dynamic displacements (provided the flow remains attached), and for the investigation of interference phenomena in this type of unsteady flows. Vortex-particle methods $[16,17]$ adopt a "vortical" interpretation of the wake, which consists of point vortices or vortex blobs, and are particularly well suited to acceleration algorithms. On the other hand, the general Unsteady Vortex-Lattice Method (UVLM) [18] represents the wake by a continuous vortex sheet, and it has been shown [19] to be a good candidate for, at least, preliminary studies of strongly interfering flow fields - the use of the UVLM has also been reported in several related disciplines 
where modeling the free-wake dynamics has a critical effect, such as rotorcraft aerodynamics [20] and air-traffic management [21].

The UVLM describes the inviscid wake by discrete vortex filaments arranged in quadrilateral vortex rings. This can create numerical difficulties when wake filaments cross trailing surfaces, due to the singularity of the Biot-Savart law, but several solution are available, such as remeshing [16], discarding wake connectivity [17, 22], or introducing a vortex-core to model the inner viscous part of the filament $[20,23,24]$. As with other potential-flow methods, the inability to account for viscous effects is one of the main limitations of the UVLM. However, different ways of overcoming it have been successfully proposed in the literature, in particular, including ad hoc stall models $[8,25]$ and using the lift-drag polar to predict viscous drag [26].

The goal of this work is to present a unified model for the aeroelastic and flight dynamics analysis of VFA, including geometrically-nonlinear deformations and, as a new feature, wake interference. For this purpose, and based on a previous assessment of different available tools [10], the general Unsteady Vortex-Lattice Method (Section II) has been coupled with a geometrically-exact composite beam finite-element model (Section III); here the primary structural variables are the local displacements and the Cartesian rotation vector, and the rigid-body dynamics of the unsupported structure are captured by the translational and angular velocities of a body-fixed reference frame. This integrated framework (Section IV) for Simulation of High Aspect Ratio Planes (SHARP) provides a modular tool to study flexible aircraft, including static aeroelastic analyses, aircraft trimming, and fully nonlinear time-marching simulations. The numerical exercises presented (Section V) will illustrate some of these capabilities, mainly focusing on the impact of wake interference on the prescribed motions and open-loop free-flight response of a representative HALE aircraft configuration.

\section{Aerodynamic Model: Unsteady Vortex-Lattice Method}

The Unsteady Vortex-Lattice Method (UVLM) is an efficient computational technique to solve 3-D potential-flow problems about moving (and deforming) lifting surfaces. The basics of the UVLM algorithm are described by Katz and Plotkin [18], and just a brief overview of our implementation is included here to make the paper self-contained. Part of this formulation might seem atypical due 
to the use of very sparse matrices, but it provides a very compact notation.

Elementary (singularity) solutions are distributed over lifting surfaces and the non-penetration boundary condition is imposed at a number of control (collocation) points, leading to a system of algebraic equations at each time step. The UVLM is based on thin wing approximation, so both the elementary solutions and the collocation points are placed over the instantaneous (deformed) mean surface in lieu of the actual surface, thus effectively ignoring thickness effects. The elementary solution is the so-called vortex ring, i.e., a quadrilateral element composed by discrete vortex segments in a closed loop, along which the circulation strength, $\Gamma_{k}$, is constant (see Fig. 1). As the surface moves following a certain flight path, a force-free inviscid wake is obtained as part of the solution procedure. The wake is represented by vortex rings, and it is formed, shed, convected and allowed to roll up according to the local flow velocity.

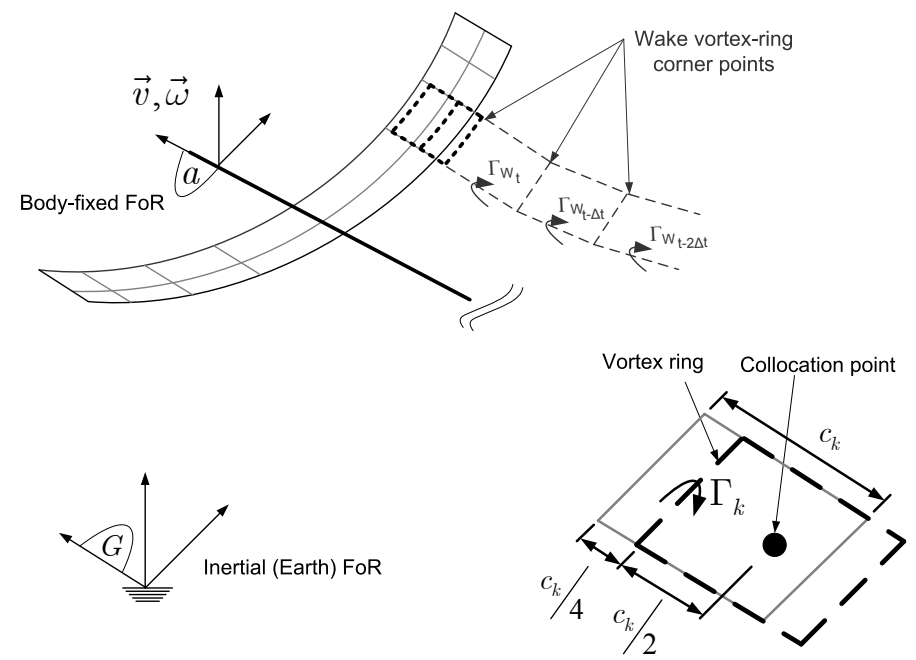

Fig. 1 Unsteady aerodynamics model: lifting surface and wake discretization using vortex-ring elements.

At discrete time step $n+1$, the non-penetration boundary condition can be formulated as

$$
A_{b} \boldsymbol{\Gamma}_{b}^{n+1}+A_{w} \boldsymbol{\Gamma}_{w}^{n+1}=\boldsymbol{w}^{n+1}
$$

where $\boldsymbol{\Gamma}_{b}$ and $\boldsymbol{\Gamma}_{w}$ are the vectors with the circulation strengths in the bound and wake vortexrings, respectively; $A_{b}=A_{b}\left(\boldsymbol{X}_{b}^{n+\epsilon}\right)$ and $A_{w}=A_{w}\left(\boldsymbol{X}_{b}^{n+\epsilon}, \boldsymbol{X}_{w}^{n+\epsilon}\right)$ are the wing-wing and wing-wake aerodynamic influence coefficient matrices, and $\boldsymbol{X}_{b}$ and $\boldsymbol{X}_{w}$ are the vectors with the bound and wake 
grid-coordinates. Elements of these matrices are obtained by projecting the velocity computed using the Biot-Savart law over the vortex-ring normal vector, and the time at which they are evaluated within the current time step, determined by $0 \leq \epsilon \leq 1$, depends on the integration scheme. The right hand side in Eq. (1) is the vector of normal components of the non-vortical velocities at the collocation points, and may include deployment of control surfaces, gust-induced velocities, wing deformations and rigid-body motions.

At each time step, a new row of vortex rings will be shed into the wake from the trailing edge of each lifting surface. In addition to this, the existing wake will displace following the local flow velocity (the free-wake model). The propagation equation for the wake circulation can be written in discrete time as

$$
\boldsymbol{\Gamma}_{w}^{n+1}=B_{b} \boldsymbol{\Gamma}_{b}^{n}+B_{w} \boldsymbol{\Gamma}_{w}^{n},
$$

where $B_{b}$ and $B_{w}$ are very sparse constant matrices which account for Kelvin's circulation theorem (that enforces the condition for wake shedding at the trailing edge) and Helmholtz's vortex theorem (in the convection of the wake). They map the wake circulation of the previous time step to the current one. As the influence of the wake decays very rapidly as it is convected away from the lifting surface (Biot-Savart law), the computational burden can be significantly alleviated by neglecting the influence of very far away panels (wake truncation). The length of wake that needs to be kept is determined through a convergence study and in this work 20 chord lengths were found to be enough for an error in the circulation vector (2-norm) of less than $1 \%$. No dissipation model $[27,28]$ has been implemented in this work.

In turn, the shedding and convection of the free wake is written as

$$
\boldsymbol{X}_{w}^{n+1}=C_{b} \boldsymbol{X}_{b}^{n+1}+C_{w} \boldsymbol{X}_{w}^{n}+\int_{t^{n}}^{t^{n+1}} \boldsymbol{V}(t) \mathrm{d} t
$$

where $C_{b}$ and $C_{w}$ in Eq. (3) are very sparse constant matrices that update the position of the prescribed wake: the former closes the newly shed wake panel with the trailing edge of the lifting surface, satisfying the Kutta condition, while the latter preserves the wake of the previous time step unchanged. The vector $\boldsymbol{V}$ in Eq. (3) includes the local (inertial) flow velocities at the grid points of the wake mesh. If a prescribed wake were to be considered, the integral term would be dropped, but 
for a fully force-free wake it is necessary to retain it and time-integration is required to determine the location of the rolled-up wake. Conventionally, this is done using an explicit one-step Euler method, but in order to improve the accuracy and/or stability of the wake rollup other higher order schemes have been also proposed in the literature, such as a two-step Euler [29] and the fourth order Adams-Bashforth-Moulton [20]. The explicit approach has been found to work well for the applications under study, and it will be used here.

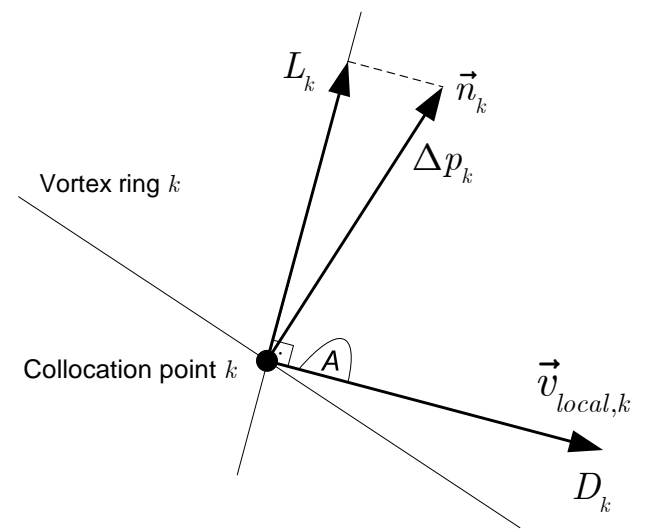

Fig. 2 Two-dimensional projection of local frame $A$ of vortex ring $k$, defined by the local instantaneous velocity at the collocation point and the normal vector.

Finally, once the distribution of vorticity has been obtained at each time step, the inviscid aerodynamic loads can be computed. The aerodynamic forces act on the plane defined by the instantaneous inertial velocity at the collocation point, and the normal vector of the vortex ring. This defines a local frame, $A$, as illustrated in the 2-D projection in Fig. 2. The pressure differential, obtained from the unsteady Bernoulli equation, acts along the normal vector. However, as the UVLM is based on thin-wing theory, it does not account for the leading-edge suction, and only the component normal to the inertial velocity is retained, i.e., the contribution of pressure to the local lift. The induced drag is aligned with the local instantaneous velocity and it is computed through an approximation to the Kutta-Joukowski theorem proposed in Ref. [18]. The vectors storing these inviscid loads are given by

$$
\begin{aligned}
\boldsymbol{L}^{n} & =\rho_{\infty} G_{c}\left[\left(U_{i} \Delta_{i}+U_{j} \Delta_{j}\right) \boldsymbol{\Gamma}_{b}^{n}+\dot{\boldsymbol{\Gamma}}_{b}^{n}\right] \\
\boldsymbol{D}^{n} & =\rho_{\infty}\left[-U^{*} \Delta_{i} \boldsymbol{\Gamma}_{b}^{n}+G_{s} \dot{\boldsymbol{\Gamma}}_{b}^{n}\right]
\end{aligned}
$$


where $\Delta_{i(j)}$ are matrices filled with 1 and -1 in the correct positions in order to account for adjacent panels; matrices $G_{c(s)}=G_{c(s)}\left(\boldsymbol{X}_{b}^{n}, \dot{\boldsymbol{X}}_{b}^{n}\right)$ are diagonal matrices dependent on the current panel geometry and local angle of incidence; $U_{i(j)}=U_{i(j)}\left(\boldsymbol{\Gamma}_{w}^{n}, \boldsymbol{X}_{b}^{n}, \boldsymbol{X}_{w}^{n}, \dot{\boldsymbol{X}}_{b}^{n}\right)$ and $U^{*}=U^{*}\left(\boldsymbol{\Gamma}_{b}^{n}, \boldsymbol{\Gamma}_{w}^{n}, \boldsymbol{X}_{b}^{n}, \boldsymbol{X}_{w}^{n}\right)$ are diagonal matrices that store weighted velocities. Their exact definitions can be found in Ref. [18].

\section{Flexible-Body Dynamics: Displacement-Based Geometrically-Exact Composite Beam}

All principal airframe structures will be modeled as composite curvilinear beams capable of large deflections and global rotations, but under the assumption of small local strains $[2,30]$. The equations of motions are solved using a finite-element discretization with nodal displacements and the Cartesian Rotation Vector (CRV) as primary degrees of freedom [11, 12]. There are no constraints on the undeformed configuration allowing the beam to be initially curved and twisted. As shown in Fig. 3, the vehicle dynamics are described by a body-fixed frame of reference (FoR), $a$, which moves with respect to an inertial frame, $G$, by the translational, $v_{a}(t)$, and angular, $\omega_{a}(t)$, velocities of its origin - subscripts are used to indicate the coordinate system in which each vector magnitude is projected. The orientation of the body-fixed frame with respect to the inertial one is given by the coordinate transformation matrix $C^{G a}(t)$, and a flat Earth is assumed. The local orientation of the beam cross sections (airfoils) is defined by their local coordinate systems, $B$, in the deformed (or current) configuration.

The equations of motion are obtained from Hamilton's principle [10], for which the potential, $\mathcal{U}$, and kinetic, $\mathcal{T}$, energy densities per unit length are first computed as

$$
\mathcal{U}=\frac{1}{2}\left\{\begin{array}{ll}
\gamma^{T} & \kappa^{T}
\end{array}\right\} \mathcal{S}\left\{\begin{array}{c}
\gamma \\
\kappa
\end{array}\right\}, \quad \text { and } \quad \mathcal{T}=\frac{1}{2}\left\{\begin{array}{ll}
V_{B}^{T} & \Omega_{B}^{T}
\end{array}\right\} \mathcal{M}\left\{\begin{array}{c}
V_{B} \\
\Omega_{B}
\end{array}\right\}
$$

Here, $V_{B}$ and $\Omega_{B}$ are the inertial velocities of the local deformed frame, $\gamma$ and $\kappa$ are the beam strains, and $\mathcal{M}$ and $\mathcal{S}$ are the mass and stiffness matrices, respectively, which are obtained through an appropriate cross-sectional analysis methodology [31]. The orientation of cross sections at each point in the current configuration is described in terms of finite rotations from the body-fixed reference frame, $a$, to the local deformed frame, $B$, using the CRV, $\Psi(s, t)$. The corresponding 


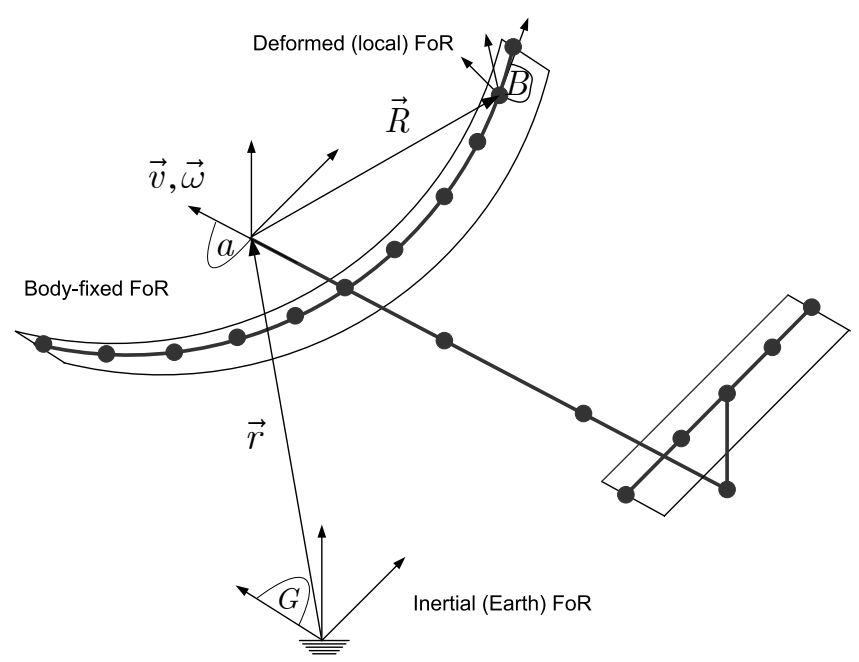

Fig. 3 Structural model: geometrically-exact beam elements.

coordinate transformation matrix will be $C^{B a}(\Psi)$. Strains and velocities can then be expressed in terms of the independent set of variables, $R_{a}(s, t)$ and $\Psi(s, t)[12,30]$,

$$
\begin{aligned}
\gamma(s, t) & =C^{B a}(\Psi(s, t)) R_{a}^{\prime}(s, t)-C^{B a}(\Psi(s, 0)) R_{a}^{\prime}(s, 0), \\
\kappa(s, t) & =T(\Psi(s, t)) \Psi^{\prime}(s, t)-T(\Psi(s, 0)) \Psi^{\prime}(s, 0), \\
V_{B}(s, t) & =C^{B a}(\Psi(s, t)) \dot{R}_{a}(s, t)+C^{B a}(\Psi(s, t))\left[\widetilde{w}_{a}(t) R_{a}(s, t)+v_{a}(t)\right], \\
\Omega_{B}(s, t) & =T(\Psi(s, t)) \dot{\Psi}(s, t)+C^{B a}(\Psi(s, t)) \omega_{a}(t),
\end{aligned}
$$

where $T(\Psi)$ is the tangential operator [12]. The forcing terms in the equations of motion require the evaluation of the position vector in the current configuration at the material points where the force is applied. From Fig. 3, and expressed in its components in the reference inertial frame it is

$$
X_{G}=r_{G}+C^{G a} R_{a}+C^{G a} C^{a B} \xi_{B}
$$

where $\xi_{B}$ contains the sectional coordinates. Taking variations in this expression the virtual displacements are obtained. If they are now multiplied by the forces and moments per unit length of the beam, as well as by their resultants on the body-fixed reference frame, one obtains the virtual work of the applied forces. This, together with the energies in Eq. (5) allows writing the equations of motion from Hamilton's principle. This general description of the beam dynamics is independent of any discretization used. Here, the position and rotation vectors within the $n^{\text {th }}$ element are approximated by given shape functions, and linear and quadratic elements have been implemented. If 
$\boldsymbol{\eta}$ is the vector of all nodal displacements and rotations, $\boldsymbol{\eta}=\left[\boldsymbol{R}_{a}^{T} \boldsymbol{\Psi}^{T}\right]^{T}$, the equations of motion can be cast into the following discrete form [10]

$$
M(\boldsymbol{\eta})\left\{\begin{array}{c}
\ddot{\boldsymbol{\eta}} \\
\dot{v}_{a} \\
\dot{\omega}_{a}
\end{array}\right\}+Q_{g y r}\left(\boldsymbol{\eta}, \dot{\boldsymbol{\eta}}, v_{a}, \omega_{a}\right)+Q_{\text {stif }}(\boldsymbol{\eta})=Q_{e x t}\left(\boldsymbol{\eta}, \dot{\boldsymbol{\eta}}, v_{a}, \omega_{a}, \zeta\right),
$$

where matrix $M$ is the tangent mass matrix and $Q_{g y r}, Q_{s t i f f}$ and $Q_{e x t}$ are the discrete gyroscopic, stiffness, and external generalized forces, respectively. Quaternions, $\zeta$, are used for the orientation of the aircraft. The linearized (incremental) form of Eq. (8) around an equilibrium point is given by

$$
M\left\{\begin{array}{c}
\Delta \ddot{\boldsymbol{\eta}} \\
\Delta \dot{v}_{a} \\
\Delta \dot{\omega}_{a}
\end{array}\right\}+C\left\{\begin{array}{c}
\Delta \dot{\boldsymbol{\eta}} \\
\Delta v_{a} \\
\Delta \omega_{a}
\end{array}\right\}+K\left\{\begin{array}{c}
\Delta \boldsymbol{\eta} \\
0 \\
0
\end{array}\right\}=\Delta Q_{e x t}\left(\Delta \boldsymbol{\eta}, \Delta \dot{\boldsymbol{\eta}}, \Delta v_{a}, \Delta \omega_{a}, \Delta \zeta\right)
$$

where $C$ and $K$ are the tangent damping and stiffness matrices, and depend on the equilibrium point.

As mentioned above, the orientation of the body-fixed reference frame with respect to the inertial frame is parameterized with quaternions, $\zeta(t)=\left(\zeta_{0}, \zeta_{v}\right)$, which need to satisfy the following propagation equation [32]

$$
\begin{aligned}
& \dot{\zeta}_{0}=-\frac{1}{2} \omega_{a}^{T} \zeta_{v}, \\
& \dot{\zeta}_{v}=\frac{1}{2}\left(\zeta_{0} \omega_{a}-\tilde{\omega}_{a} \zeta_{v}\right) .
\end{aligned}
$$

The instantaneous coordinate transformation matrix $C^{G a}$ and position vector of the body-fixed reference frame are finally obtained as

$$
\begin{aligned}
C^{G a} & =\left(2 \zeta_{0}^{2}-1\right) I+2\left(\zeta_{v} \zeta_{v}^{T}+\zeta_{0} \tilde{\zeta}_{v}\right), \\
\dot{r}_{G} & =C^{G a} v_{a} .
\end{aligned}
$$

\section{Coupled Aeroelasticity and Flight Dynamics of Flexible Aircraft}

The previous unsteady aerodynamic and flexible-body models will be used to represent the complete dynamics of a flexible air vehicle. As the structural model is based on beams (curves in space) and the aerodynamic lattice is distributed over a lifting surface, a mapping procedure is 
required between both meshes, and how this is tackled is presented in Sections IV A and IV B. The different solution methodologies that will be used for the numerical results are outlined in Section IV C.

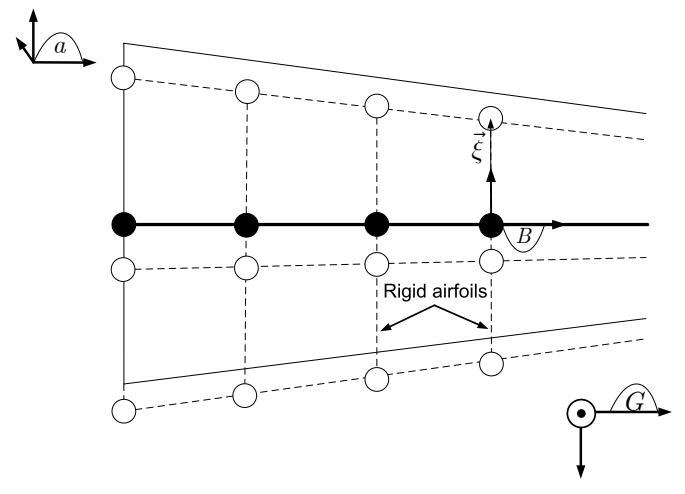

(a) Mapping between beam nodes and aerodynamic grid. A discontinuous line is used for the vortex rings and a continuous line for the beam. Aerodynamic corner points are hollow and beam nodes are solid.

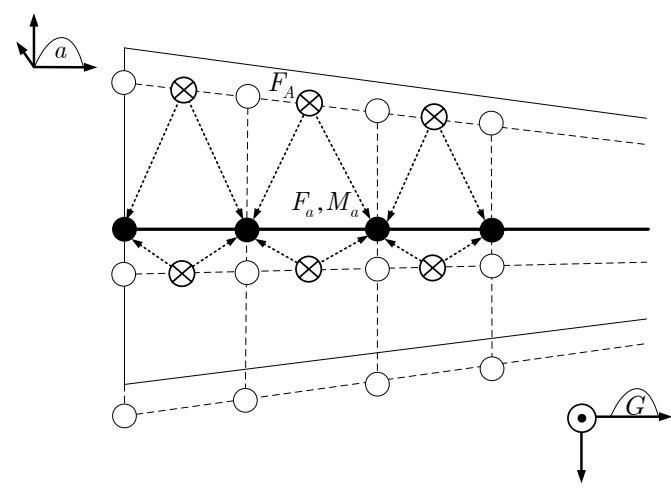

(b) Mapping of inviscid aerodynamic forces, $F_{A}$, to nodal forces and moments $\left(F_{a}, M_{a}\right)$. Locations at which aerodynamic forces act (center of vortex-ring leading segment) are marked with crosses.

Fig. 4 Mapping between aerodynamic lattice and structural finite-element discretization: (a) geometry, and (b) aerodynamic loads.

\section{A. Mapping Structural Displacements and Velocities to the Aerodynamic Model}

Firstly, displacements and rotations of the beam nodes, $\boldsymbol{R}_{a}$ and $\boldsymbol{\Psi}$, and the corresponding rates, $\dot{\boldsymbol{R}}_{a}$ and $\dot{\boldsymbol{\Psi}}$, have to be transformed to deformations and velocities of the grid points of the aerodynamic lattice, $\boldsymbol{X}_{b}$ and $\dot{\boldsymbol{X}}_{b}$. This is done assuming airfoil sections remain rigid under wing deformations. Vortex-ring corner points and collocation points are expressed in the lifting surface coordinate system, which is rigidly linked to the body-fixed one, $a$. In the initial (undeformed) configuration a mapping between the structural nodes and the aerodynamic grid can be defined, as illustrated in Fig. 4(a). For the sake of simplicity, the finite-element discretization of the beam coincides with the spanwise aerodynamic grid, but cambered airfoils are allowed. The distance between a vortex-ring corner point and the relevant node, $\xi_{B}$, will remain constant, and as a consequence, it is possible to determine the aerodynamic grid in the body-fixed FoR, at any deformed configuration of the member. For each vortex-ring corner point the following transformation is 
defined (variables are not bold since the transformation corresponds to a single vortex-ring corner point)

$$
\left(X_{b}\right)_{a}=R_{a}+C^{a B}(\Psi) \xi_{B}
$$

The transformation for the inertial velocities is

$$
\left(\dot{X}_{b}\right)_{a}=v_{a}+\widetilde{\omega}_{a} R_{a}+\dot{R}_{a}+C^{a B}(\Psi) \widetilde{\Omega}_{B} \xi_{B}
$$

where the local inertial angular velocity, $\Omega_{B}^{n}$, was given in Eq. (6). Positions and velocities of the collocation points are obtained through interpolation.

\section{B. Mapping Aerodynamic Forces to the Structural Model}

To transform the aerodynamic loads computed in Eqs. (4) to forces and moments acting upon the beam nodes it is assumed that they are point forces applied at the center of the leading segment of each vortex ring. Each vortex ring has a local $A$ frame associated, defined by the local instantaneous velocity, as explained in Section II. The inviscid aerodynamic forces at vortex ring $k$, expressed in the local aerodynamic frame, $A$, are given by

$$
\left(F_{k}\right)_{A}=\left[\begin{array}{lll}
D_{k} & 0 & L_{k}
\end{array}\right]^{T}
$$

with $L_{k}$ and $D_{k}$ the lift and the induced drag of panel $k$, as given by Eqs. (4). These forces are then transformed to the body-fixed $a$ frame, in order to be consistent with the flexible-beam equations, Eqs. (8-10). Finally, they are lumped into the nodes of the deformed beam, splitting them between adjacent ones as illustrated in Fig. 4(b) - note that this mapping will give rise to moments acting at the corresponding nodes. These operations can be summarized as

$$
\left\{\begin{array}{l}
\boldsymbol{F}_{a} \\
\boldsymbol{M}_{a}
\end{array}\right\}=\chi \bar{C}^{a A} \boldsymbol{F}_{A},
$$

where $\bar{C}^{a A}\left(\boldsymbol{X}_{b}, \dot{\boldsymbol{X}}_{b}\right)$ is a block diagonal matrix, being each block given by the corresponding coordinate transformation matrix from the local aerodynamic to the body-fixed frame, $C^{a A}$. In turn, $\chi=\chi\left(\boldsymbol{R}_{a}, \Psi, \boldsymbol{X}_{b}\right)$ is a very sparse matrix that lumps the forces acting on the aerodynamic lattice, expressed in $a$, into forces and moments applied on the beam nodes. Integration of these nodal values yields the resultant forces and moments on the body-fixed FoR. 


\section{Solution Methods}

Through the above mapping procedures the coupling of aerodynamic and structural models for a full aeroelastic and flight dynamics characterization of the flexible vehicle can be tackled. The flexible-body dynamics equations, Eqs. (8-10) are integrated with the unsteady aerodynamics, Eqs. (1-4). Other external forces, in particular, gravity forces, can also be introduced into the equations of motion. Different solution approaches have been defined: static aeroelastic, trim, and open-loop dynamic simulations, including linearized and geometrically-nonlinear solutions, when appropriate. In particular, for time-domain analyses the second order continuous-time flexible-body equations are discretized using the Newmark- $\beta$ method [12], and a loosely coupled approach solves them together with the discrete-time UVLM formulation. This is a partitioned time-marching scheme [33] in which information is exchanged at each time step but no subiterations are included. The main steps of the process are:

1. Based on the geometry and velocities at time step $n$, the aerodynamic loads are computed.

2. These aerodynamic loads are applied on the flexible-body equations, which are solved using the Newmark- $\beta$ scheme in order to obtain the geometry and velocities at time step $n+1$.

3. The procedure is repeated from 1 , substituting $n+1$ instead of $n$.

The methods described above have been implemented in a framework for Simulation of High Aspect Ratio Planes. SHARP is built on a modular architecture in Matlab, but with low-level libraries in Fortran, that allows running independently the flexible-body dynamics and aerodynamics solvers, as well as the coupled system. As remarked above, the flexible-beam equations are geometrically exact, and therefore capture the nonlinearities that arise due to large deformations, updating the relevant inertia, gyroscopic and stiffness terms. Even though the vortex-lattice method used for the unsteady aerodynamics is based on potential-flow theory, the boundary conditions are enforced at the deformed shape, thus accounting for large motions of the lifting surfaces. Aerodynamic control surfaces are directly modeled by prescribed deflections of trailing edge panels and the true shape of the wake can be obtained as part of the solution procedure - however, a prescribed-wake model is often a good approximation and it has also been implemented. 


\section{Numerical studies}

This section presents several numerical studies using SHARP. Verification of the structuraldynamics solution under given loads, as well as unsteady aerodynamics with prescribed kinematics, have been presented in a previous paper [10], and will not be repeated here. The implementation of the coupled model is verified computing the flutter speed of the Goland wing (Section V A), and comparing SHARP to published results for the large static deformations of a representative HALE flexible aircraft (Section VB). Next, interference effects are analyzed for the prescribed longitudinal motions of the HALE vehicle, investigating proximity effects (Section V C), and briefly discussing direct wake-tail collisions (Section VD). Finally, the influence of aerodynamic interference is assessed on the open-loop response of the free-flying aircraft (Section VE).

\section{A. Linear stability analysis of the Goland wing}

The Goland wing is a stiff and small aspect ratio cantilever wing that is often used for verification purposes. The relevant properties can be found, for instance, in Ref. [2]). Here, its flutter speed has been estimated in the time domain, assuming the air density to be $\rho_{\infty}=1.020 \mathrm{~kg} / \mathrm{m}^{3}$. The clamped wing is started from rest with a very small angle of attack of $\alpha=0.05 \mathrm{deg}$, and the coupled aeroelastic model is marched in time in a linear dynamic simulation (without rigid-body degrees of freedom). Different free-stream velocities are evaluated until the flutter onset is found.

Fig. 5 depicts the time-histories of the wing-tip deflection at different air speeds near the flutter onset. Examples of decaying $\left(V_{\infty}=160 \mathrm{~m} / \mathrm{s}\right)$ and growing $\left(V_{\infty}=170 \mathrm{~m} / \mathrm{s}\right)$ periodic responses are presented together with the neutral speed $\left(V_{f}=165 \mathrm{~m} / \mathrm{s}\right)$. Instability is due to a bending-torsion coupling, and the flutter frequency is $\omega_{f}=69 \mathrm{rad} / \mathrm{s}$.

Table 1 compares the flutter velocity and angular frequency obtained with SHARP to results found in the literature. Goland [34, 35] obtained results using an analytical beam model with 2-D aerodynamics. It is worth noting that the original results presented in Ref. [34] were erroneous, and they were later corrected in Ref. [35]. Only the correct values are shown in Table 1, and good agreement with other models based on strip theory can be seen. Interestingly, while the original (incorrect) values for the Goland-wing flutter were accidentally closer to the results computed via 


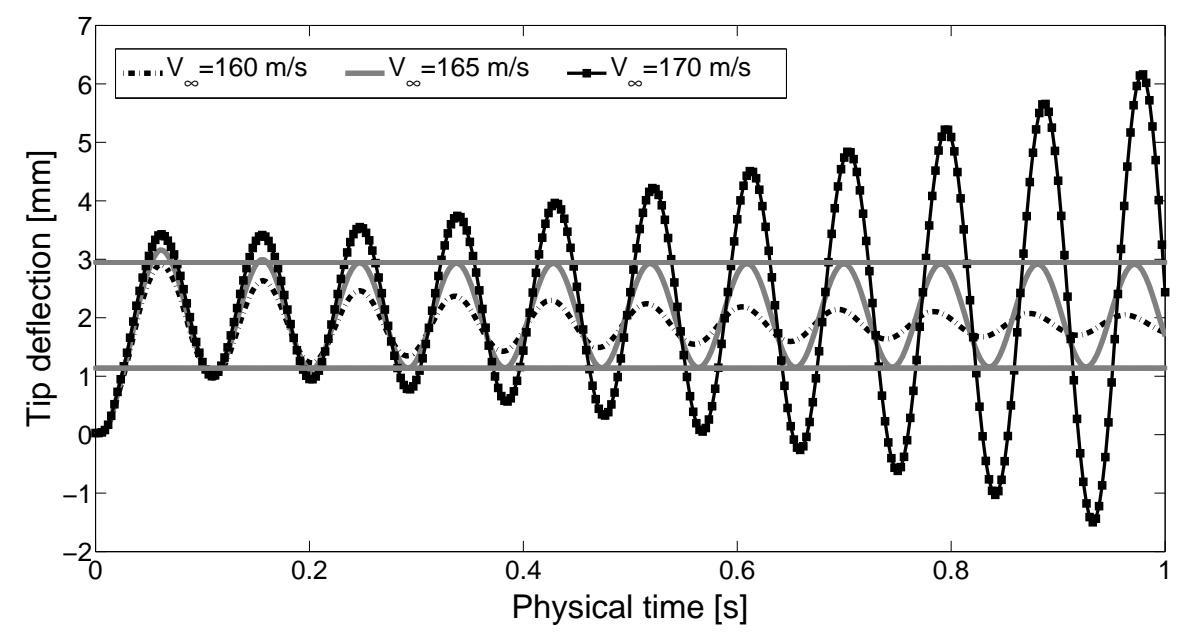

Fig. 5 Goland wing-tip time-history at various free-stream velocities.

the UVLM [36], the correct ones exhibit a substantial disagreement, which shows the importance of 3-D aerodynamic effects. The small discrepancies that can be observed between the current implementation and other three-dimensional aerodynamic models are within the expected range.

Table 1 Flutter speed of the Goland wing

\begin{tabular}{lllc}
\hline \hline Author & Model & $V_{f}[\mathrm{~m} / \mathrm{s}]$ & $\omega_{f}[\mathrm{rad} / \mathrm{s}]$ \\
\hline Goland [35] & Analytical & 137.2 & 70.7 \\
Patil [2] & Intrinsic beam + strip theory & 135.6 & 70.2 \\
Wang et al. [36] & ZAERO [37] & 174.3 & - \\
Wang et al. [36] & Intrinsic beam + UVLM & 163.8 & - \\
SHARP & Displacement beam + UVLM & 165 & 69 \\
\hline \hline
\end{tabular}

\section{B. Nonlinear static aeroelasticity of flexible aircraft}

The nonlinear aeroelastic solver is assessed next. For that purpose, a representative HALE aircraft has been defined, based on the one proposed by Patil and co-workers [3]. The vehicle consists of a large aspect ratio flexible wing, a rigid fuselage and and a rigid tail that includes a 25\%-chord elevator. All lifting surfaces are untwisted, unswept, and untapered. The aircraft carries a payload of $50 \mathrm{~kg}$ in the fuselage (a point mass at a distance $d_{p l}$ from the wing elastic-axis) and it is powered by two propellers, which are modeled as point follower-forces at the elastic axis. The 
geometry of the aircraft is shown in Fig. 6 and the relevant properties are listed in Table 2. It will be assumed that the aircraft flies at an altitude of $20 \mathrm{~km}$, where the density is $\rho_{\infty}=0.0899$ $\mathrm{kg} / \mathrm{m}^{3}$. This aircraft will be used for all subsequent results, and as it can be observed, the stiffness properties of the main wing, and the locations of the payload, $d_{p l}$, and the horizontal tail plane $(\mathrm{HTP}), d_{H T P}$, will be varied as parameters.
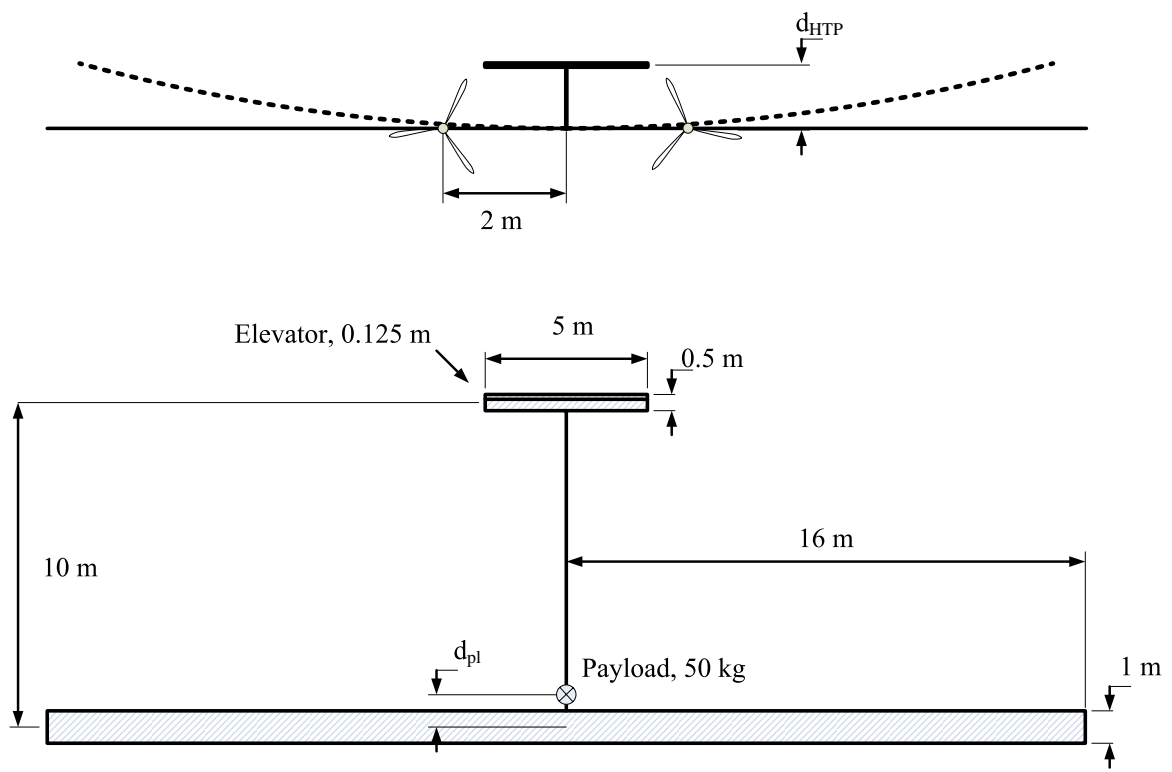

Fig. 6 HALE model aircraft geometry. Large aspect ratio straight wing, rigid fuselage and tail, and propellers (not to scale). Front and top views, showing typical wing deformation.

In the first instance, SHARP is compared to the static aeroelastic results obtained by Smith et al. [38] using higher-fidelity aerodynamic modeling. Those authors modeled the large deflections of a cantilever wing coupling a nonlinear beam model [30] with a three-dimensional Euler solver (ENS3DAE) [39]. The configuration corresponds to the clamped main wing of the aircraft of Fig. 6, without payload, and with $\sigma_{1}=1$ and $\sigma_{2}=5$. For the CFD results in Ref. [38] a NACA0012 airfoil was considered from root to tip. The UVLM ignores thickness and only the mean surface is modeled here. Fig. 7 displays the deflections experienced by the wing at a free-stream speed of $V_{\infty}=25 \mathrm{~m} / \mathrm{s}$, for angles of attack of $\alpha=2 \mathrm{deg}$ and $\alpha=4 \mathrm{deg}$. It can be clearly inferred that deformations are geometrically nonlinear, with tip deflections of $20 \%$ and $34 \%$ of the semi-span, respectively. The agreement with the CFD results is very good, with a maximum discrepancy of 


\begin{tabular}{lll}
\hline \hline & Main wing & Tail \\
\hline Chord & $1 \mathrm{~m}$ & $0.5 \mathrm{~m}$ \\
Semi-span & $16 \mathrm{~m}$ & $2.5 \mathrm{~m}$ \\
Elastic axis & $50 \%$ chord & $50 \%$ chord \\
Center of gravity & $50 \%$ chord & $50 \%$ chord \\
Mass per unit length & $0.75 \mathrm{~kg} / \mathrm{m}$ & $0.08 \mathrm{~kg} / \mathrm{m}$ \\
Moment of inertia (around e.a.) & $0.1 \mathrm{~kg} \cdot \mathrm{m}$ & $0.01 \mathrm{~kg} \cdot \mathrm{m}$ \\
Torsional stiffness & $\sigma_{1} \times 10^{4} \mathrm{~N} \cdot \mathrm{m}^{2}$ & $\infty$ \\
Bending stiffness & $2 \sigma_{1} \times 10^{4} \mathrm{~N} \cdot \mathrm{m}^{2}$ & $\infty$ \\
Chordwise bending stiffness & $\sigma_{2} \times 10^{6} \mathrm{~N} \cdot \mathrm{m}^{2}$ & $\infty$ \\
\hline \hline
\end{tabular}

less than $5 \%$ at the tip for $\alpha=4 \mathrm{deg}$, which can be attributed both to the different lift-curve slope between both models and details on the modeling of the wing-tip geometry.

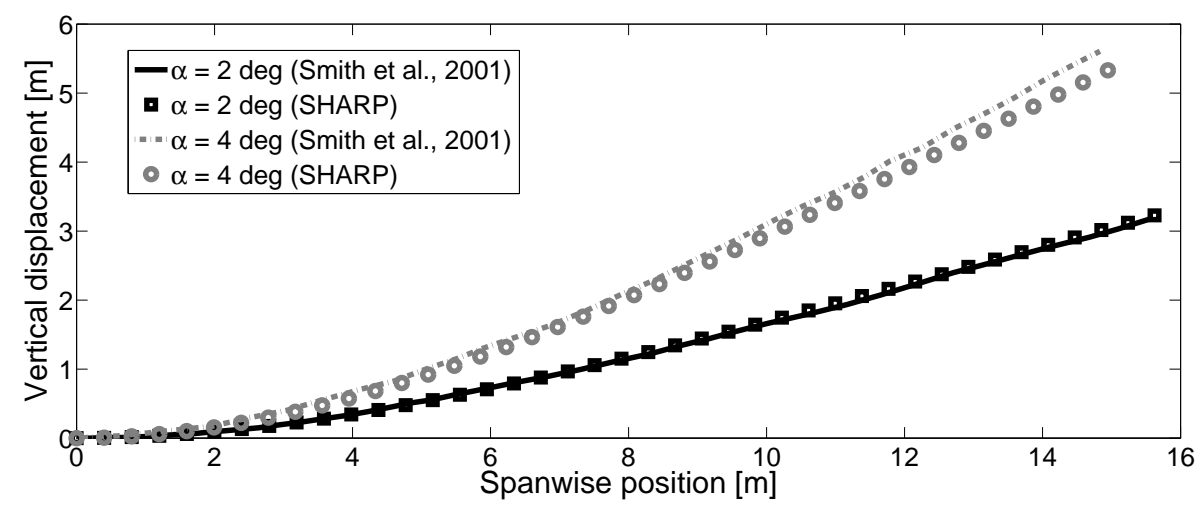

Fig. 7 Static deflections of the clamped cantilever main wing at different angles of attack. $V_{\infty}=25 \mathrm{~m} / \mathrm{s}, \sigma_{1}=1, \sigma_{2}=5$.

Next, the HALE platform will also be used to compare results with lower fidelity 2-D aerodynamic modeling. Patil et al. [3] analyzed this vehicle using the nonlinear beam equations of Hodges [30] and Peters' 2-D finite-state air-loads model [40]. In this case, $d_{p l}=2 \mathrm{~m}$ and $d_{H T P}=0 \mathrm{~m}$. The rigid and fully flexible aircraft are studied, and for the latter, $\sigma_{1}=1$ and $\sigma_{2}=4$.

Following the same time-domain solution process described in Section V A for the Goland wing, the flutter speed and frequency of the undeformed HALE aircraft described above have been found 
to be $V_{f}=33 \mathrm{~m} / \mathrm{s}$ and $\omega_{f}=22 \mathrm{rad} / \mathrm{s}$. This is in excellent agreement with the values reported in Ref. [3], $V_{f}=32.21 \mathrm{~m} / \mathrm{s}$ and $\omega_{f}=22.61 \mathrm{rad} / \mathrm{s}$ respectively, which serves to verify the current implementation for small wing oscillations. Note that, as in Ref. [3], the flutter point is obtained on perturbations on the undeformed configuration, i.e., the aircraft is not trimmed for the flutter speed.

Finally, Fig. 8 presents nonlinear static aeroelastic equilibrium for the full vehicle. Fig. $8(a)$ shows the angle of attack that produces a vertical force that balances the total weight at various flight speeds (with no elevator deflection). For the flight speeds and angles of attack investigated, the flexible wing exhibits very large tip deflections, between 35 and $50 \%$ of the semi-span. Even though the qualitative trend is the same, SHARP yields higher angles of attack in both cases, with the discrepancy being especially pronounced in the flexible case. Fig. $8(\mathrm{~b})$ depicts the total lift ratio between flexible and rigid aircraft at various free-stream velocities - the contribution of the tail to the total lift is included. In this case, a constant angle of attack has been prescribed, $\alpha=5 \mathrm{deg}$, and linear and nonlinear computations have been carried out. The mismatch between estimations clearly increases with velocity, which for a fixed angle of attack entails larger deformations.

To sum up, the present implementation has been found to agree well with higher fidelity CFD for notably nonlinear situations, and with lower fidelity 2-D aerodynamics in the linear regime. However, there is a substantial discrepancy with the latter for large wing deflections. This indicates that the tip corrections to 2-D aerodynamic models, based on the exact spanwise lift distribution of the undeformed shape, may not be appropriate for large wing bending displacements. Similar results have also been reported by Wang et al. [8] in their comparison of UVLM and 2-D aerodynamics to compute the trim angle of a very flexible flying wing.

\section{Wake proximity effects on prescribed aircraft motions}

In this section, wake-tail proximity effects are studied for prescribed longitudinal motions of the body-fixed frame, $a$. Hence, only the aeroelastic system is considered, but including the inertia forces due to the prescribed motions. The HALE aircraft described above (see Table 2 and Fig. 6 ) is considered again as test case. To simplify the problem the vertical fin is modeled as a simple beam 


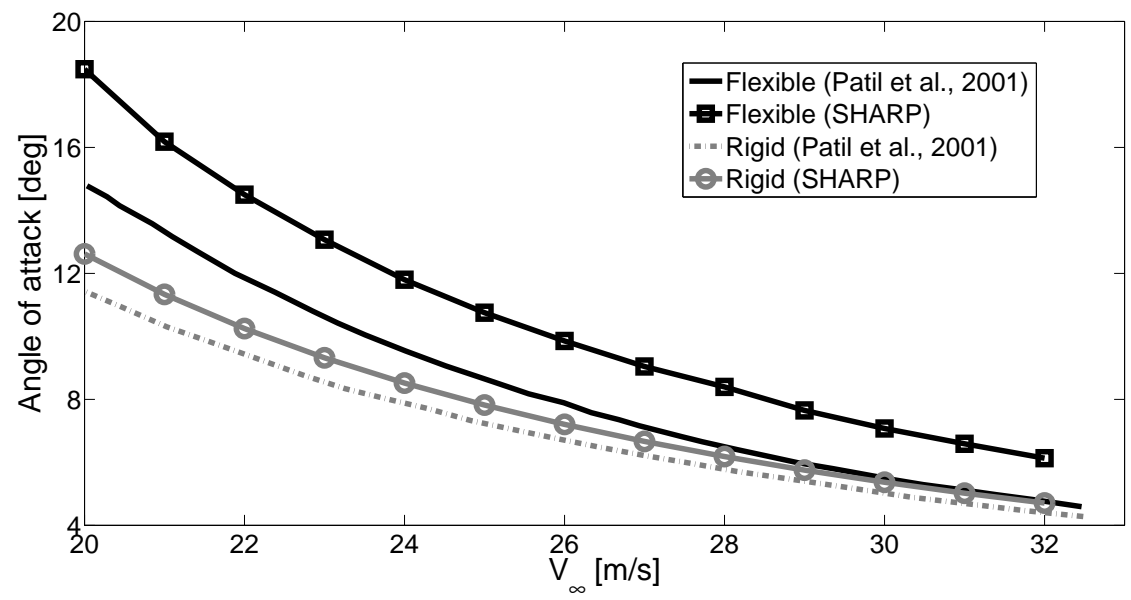

(a) Variation of angle of attack with flight speed for vertical force equilibrium.

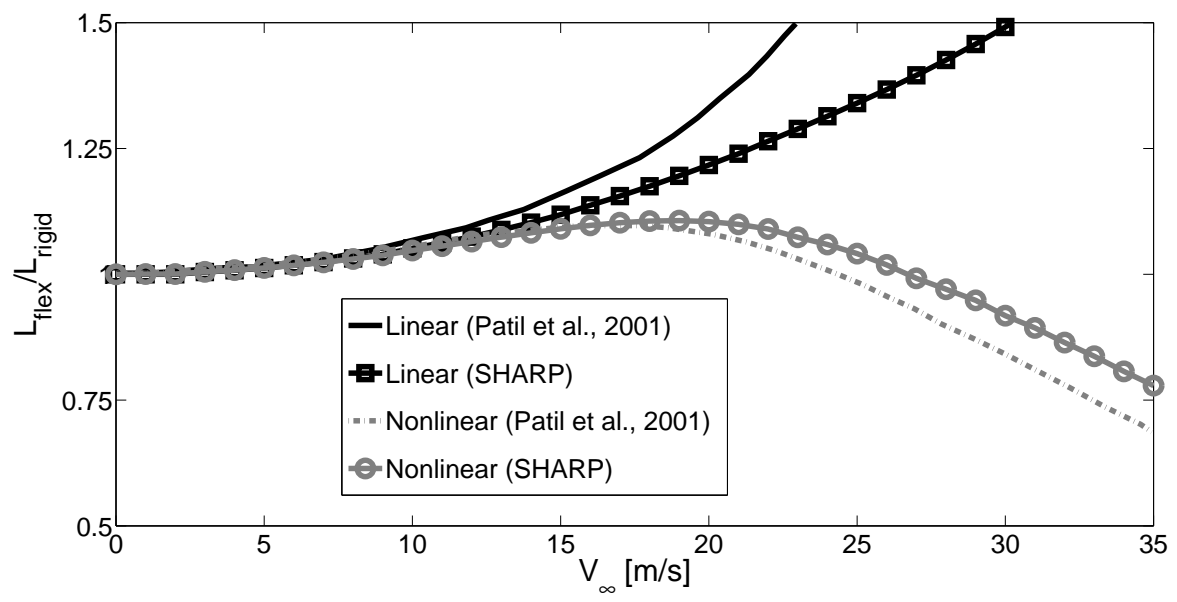

(b) Ratio of total lift of the flexible to the rigid aircraft at $\alpha=5 \mathrm{deg}$.

Fig. 8 Static results for the model HALE aircraft. $\sigma_{1}=1, \sigma_{2}=4, d_{p l}=2 \mathbf{m}, d_{H T P}=0 \mathbf{m}$.

without aerodynamic effects, and the following values have been assumed: $d_{p l}=1 \mathrm{~m}, d_{H T P}=2 \mathrm{~m}$, $\sigma_{1}=5$ and $\sigma_{2}=20$. A stiffened version of the main wing has been considered in order to focus on the interference effects. The elevator deflection is set to zero and the angle of attack of the aircraft, $\alpha_{0}$, that balances the weight is determined at the desired flight velocity. Under these conditions, the tip deflections of the main wing are of the order of $10 \%$ of the semi-span at static equilibrium. In order to investigate the influence of the wake shed by the main wing over the horizontal stabilizer three types of motion are prescribed around the corresponding equilibrium (deformed) configuration: 
plunging, pitching, and combined plunging-pitching for a constant incidence angle. That is,

$$
\begin{aligned}
& h=A_{h} \sin \left(\omega_{h} t\right), \\
& \alpha=\alpha_{0}+A_{\alpha} \cos \left(\omega_{\alpha} t\right) .
\end{aligned}
$$

First of all, a pure harmonic plunging motion of the HALE model has been analyzed, i.e., $A_{\alpha}=0$ deg. The flight velocity has been chosen to be $V_{\infty}=40 \mathrm{~m} / \mathrm{s}$, well below the flutter speed for this set of parameters, $V_{f}=74 \mathrm{~m} / \mathrm{s}$. The frequency of the oscillation is selected so that it is close to the $1^{\text {st }}$ bending mode of the main wing, $\omega_{b 1}=5.1 \mathrm{rad} / \mathrm{s}$, and has been set to $\omega_{h}=5 \mathrm{rad} / \mathrm{s}$. The amplitude has been chosen so that it coincides with the static equilibrium vertical position of the center of gravity of the main wing, and for $V_{\infty}=40 \mathrm{~m} / \mathrm{s}$, this corresponds to $A_{h}=0.79 \mathrm{~m}$. The angle of attack that balances total lift and weight at this velocity is $\alpha_{0}=2.5 \mathrm{deg}$.

The evolution of the tail lift coefficient has been monitored. The results obtained using the present coupled aeroelastic model are compared to other approximations to the tail lift coefficient slope, $C_{L, \alpha}$. The first simplification is to assume that the HTP is a wing flying with a steady wake behind, represented by horseshoe vortices (it will be referred to as the "flying tail" approximation). The second, more sophisticated, approach is to consider the whole aircraft in steady flight at the equilibrium conditions, accounting for both the wake shed by the deformed main wing and the HTP itself ("full aircraft" approximation). For $V_{\infty}=40 \mathrm{~m} / \mathrm{s}$, these approximate values for the tail lift coefficient slope are given by

$$
\begin{gathered}
\left.C_{L, \alpha}\right|_{\text {flying tail }}=4.93 \mathrm{rad}^{-1}, \\
\left.C_{L, \alpha}\right|_{\text {full A/C }}=4.58 \mathrm{rad}^{-1},
\end{gathered}
$$

where the downwash caused by the steady wake of the main wing on the HTP lift is apparent.

Fig. 9 shows the lift coefficient of the tail-plane, as a function of time and vertical displacement, for a plunging cycle after the initial transient has elapsed. Results obtained with SHARP are presented, including a free wake and for an implementation in which the rollup of the wake is switched off (prescribed wake solution). These are compared to the two different approximations described above - note that for this motion, the approximate lift coefficient is given by $C_{L, t a i l}(t)=$ $C_{L, \alpha}\left\{\alpha_{0}-\tan ^{-1}\left[A_{h} \omega_{h} \cos \left(\omega_{h} t\right) / V_{\infty}\right]\right\}$. It can be seen that the more elaborate approximation 


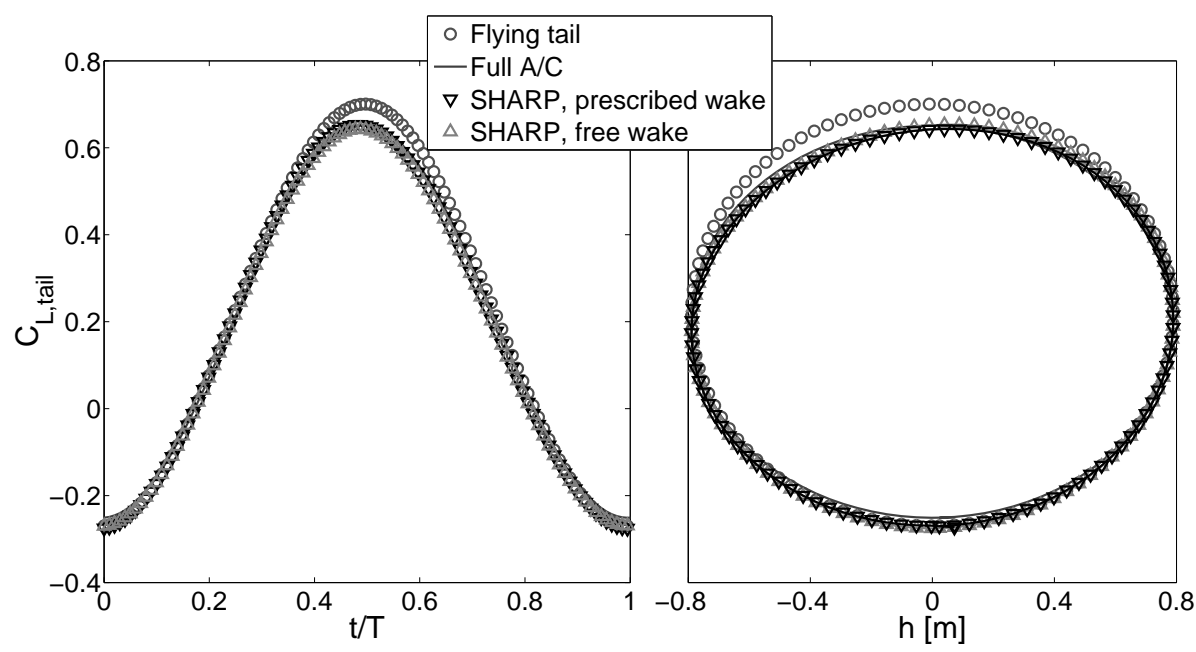

Fig. 9 Tail lift coefficient for an aircraft in plunging motion. $V_{\infty}=40 \mathrm{~m} / \mathrm{s}, \alpha_{0}=2.5 \mathrm{deg}$, $A_{h}=0.79 \mathbf{m}, \omega_{h}=5 \mathrm{rad} / \mathbf{s}, A_{\alpha}=0 \mathrm{deg}$.

provides a satisfactory agreement with respect to the solution of the coupled model, since the tail lift is mainly dominated by the prescribed plunging. In contrast, the flying tail approximation overestimates the maximum value of lift when the aircraft is descending and the wake nears the HTP, since the presence of the wake of the main wing is not taken into account. Clearly, for this particular configuration and flight conditions neglecting the wake rollup does not affect the tail lift coefficient.

The next prescribed motion subject to study is a pure harmonic pitching, i.e., $A_{h}=0$ m. This pitching motion around the equilibrium condition has been defined so that it would cancel out the induced angle of attack due to the plunging motion described above, yielding $\omega_{\alpha}=5 \mathrm{rad} / \mathrm{s}$ and $A_{\alpha}=5.6 \mathrm{deg}$. Results for this case are presented in Fig. 10, where the lift coefficient of the tail is plotted against time and pitching amplitude. Analogous disagreement to the plunging case is observed for the flying tail estimation. The full aircraft approximation again captures relatively well the oscillation amplitude, but as opposed to the pure plunging, it fails to predict the correct phase lag. The hysteresis loop present in the fully unsteady results is not predicted by any of the approximations, and hence, the interference is manifested here as an alteration of the lag between motion and loads, which quasi-steady aerodynamics models neglect.

The last motion considered is a combination of plunging and pitching, given by Eqs. (16-17), 

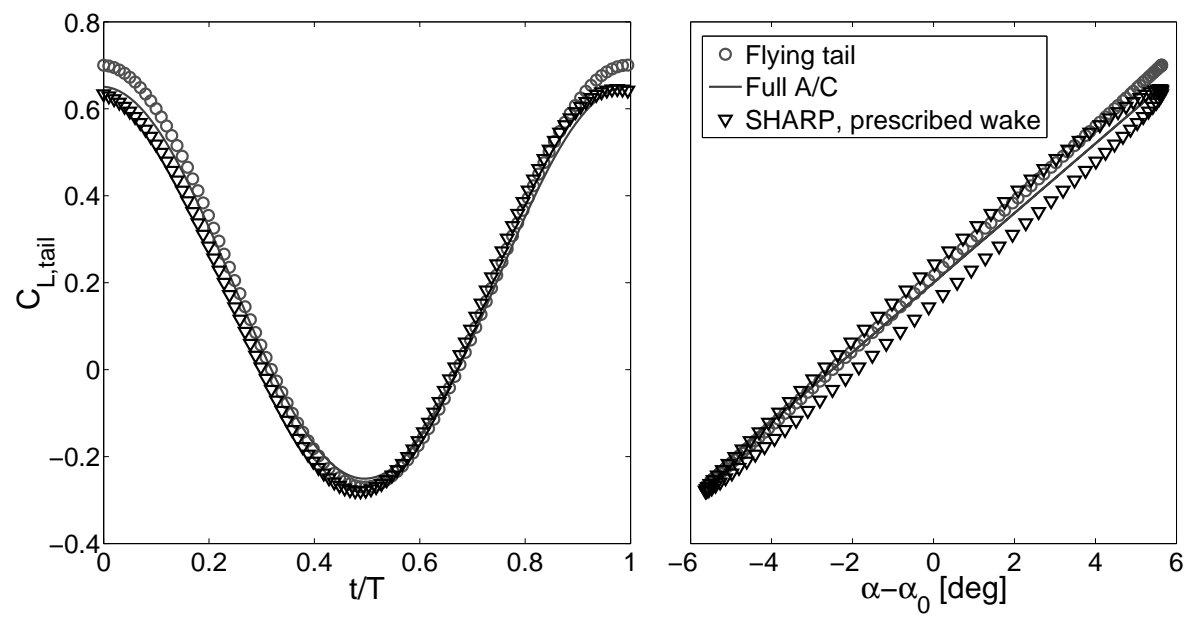

Fig. 10 Tail lift coefficient for an aircraft in pitching motion. $V_{\infty}=40 \mathrm{~m} / \mathrm{s}, \alpha_{0}=2.5 \mathrm{deg}$, $A_{\alpha}=5.6 \mathrm{deg}, \omega_{\alpha}=5 \mathrm{rad} / \mathrm{s}, A_{h}=0 \mathrm{~m}$.

using the same parameters as before. As a consequence of retaining the amplitudes and frequencies from previous cases, the effective incidence angle remains constant at the static equilibrium condition throughout the flight path, and this motion is representative of the aircraft entering a neutrally stable phugoid mode.
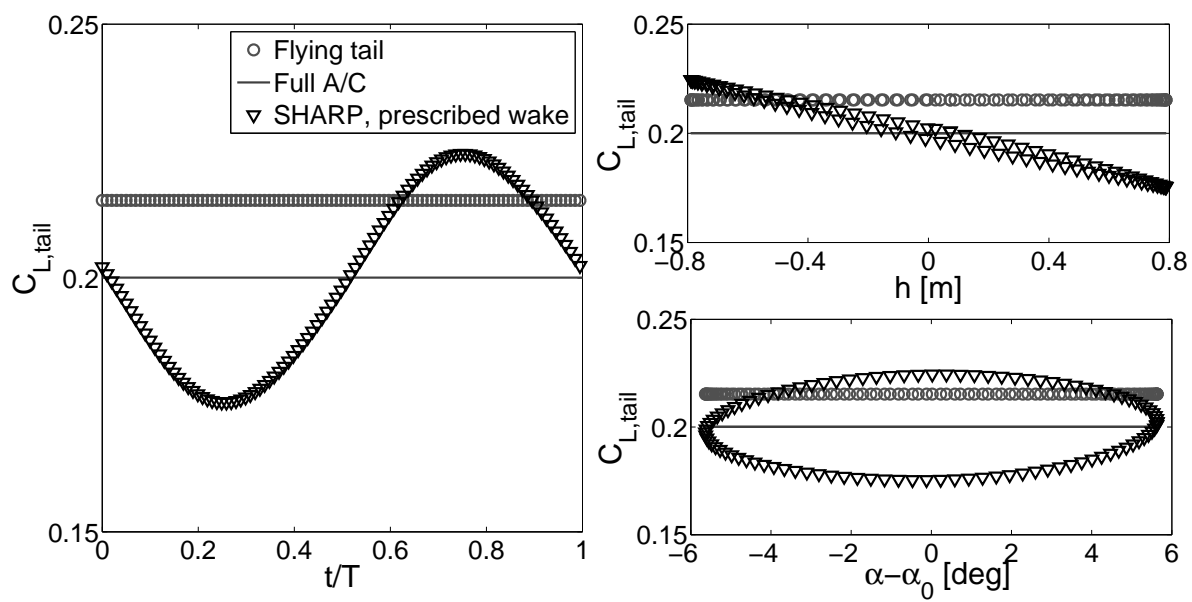

Fig. 11 Tail lift coefficient for an aircraft in a plunging and pitching combined motion. $V_{\infty}=40$ $\mathbf{m} / \mathbf{s}, \alpha_{0}=2.5 \mathrm{deg}, A_{h}=0.79 \mathbf{m}, A_{\alpha}=5.6 \mathrm{deg}, \omega_{h}=\omega_{\alpha}=5 \mathrm{rad} / \mathbf{s}$.

Results are displayed in Fig. 11. The lift coefficient of the tail is plotted against time, vertical motion, and pitching motion. Results exhibit substantial disagreements between the fully coupled model and the quasi-steady estimations. As the effective incidence angle remains constant, the 
proposed approximations predict a constant lift coefficient. In contrast, the UVLM predicts a harmonic value, consistent with the oscillatory nature of the wake, which approaches and moves away from the tail in a cyclic fashion. The full aircraft approximations gives a good estimate of the average value, and the flying tail approximation is far off due to the missing downwash. Even though the amplitude of the oscillations of the lift coefficient are not as large as in the pure plunging and pitching cases (only of the order of $10 \%$ of the average value), this might impact the longitudinal motions of the aircraft. This will be investigated in Section VE.

\section{Wake-tail impingement on prescribed aircraft motions}

The numerical characteristics of potential direct wake-empennage encounters are briefly discussed now. For that purpose, a different flight speed has been chosen for the same aircraft, $V_{\infty}=25$ $\mathrm{m} / \mathrm{s}$. In order to prescribe a purely plunging motion analogous to that in Section VC, it is $\alpha_{0}=7.2$ $\operatorname{deg}, A_{h}=0.75 \mathrm{~m}$, and $\omega_{h}=5 \mathrm{rad} / \mathrm{s}\left(\right.$ with $\left.A_{\alpha}=0 \mathrm{deg}\right)$.

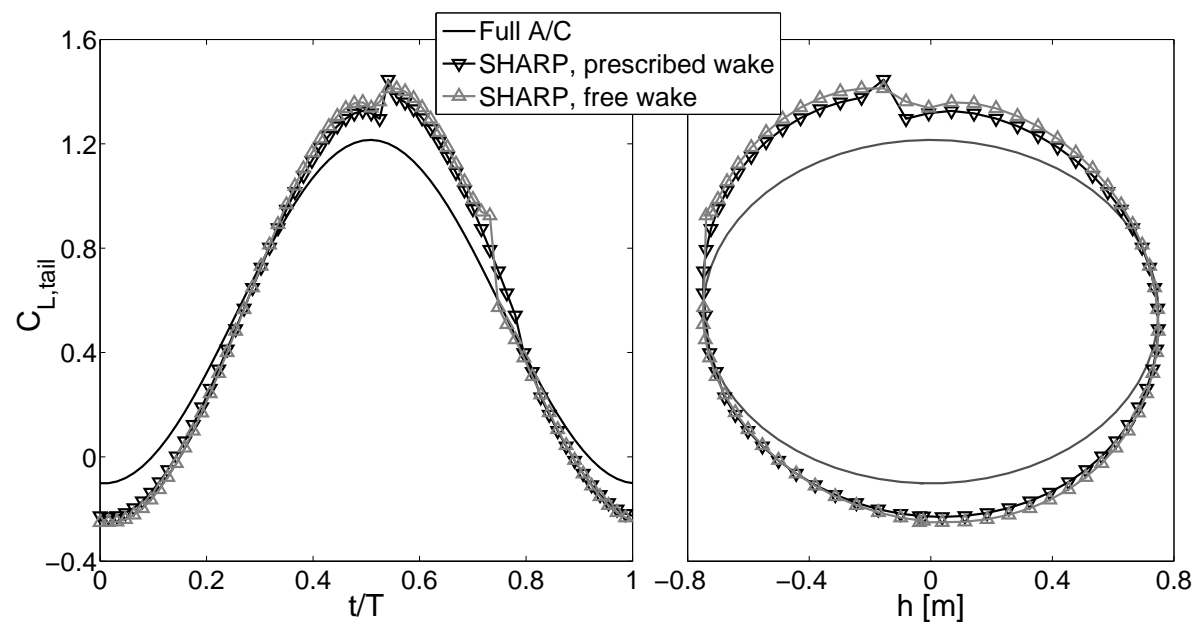

Fig. 12 Tail lift coefficient for an aircraft in plunging motion. $V_{\infty}=25 \mathrm{~m} / \mathbf{s}, \alpha_{0}=7.2 \mathrm{deg}$, $A_{h}=0.75 \mathrm{~m}, \omega_{h}=5 \mathrm{rad} / \mathrm{s}, A_{\alpha}=0 \mathrm{deg}$.

Fig. 12 shows results for this case, comparing SHARP with and without free-wake rollup, and the full aircraft approximation $\left(\left.C_{L, \alpha}\right|_{\text {full A/C }}=4.43 \mathrm{rad}^{-1}\right.$ in this case). The most notable feature is the presence of discontinuities in the values obtained through the coupled aeroelastic model, one near maximum lift, and the other at around $t / T=0.75$, caused by the tail crossing the wake shed 
by the main wing. These sudden changes experienced by the tail lift are representative examples of wake-airfoil collisions, similar to parallel Blade-Vortex Interactions (BVI) in helicopter rotors $[20,23,41-43]$. Even though it is beyond the scope of this paper to study the specific features of the vortex-body impingement in detail, the UVLM provides a first estimate of the effect of this interference [19]. Observing the first jump in Fig. 12 (near maximum lift), it can be inferred that there is a gradual decrease in lift as the wake approaches the trailing HTP, followed by a rapid rise when the nearest position is reached, which is consistent with the BVI behavior [41, 43]. It has been reported that the sudden change in lift is of the order of the nondimensional circulation strength, $\Gamma /\left(V_{\infty} c\right)$, of the impinging vortex [44], and this is roughly predicted by the UVLM. However, to the best of the authors' knowledge, there are no conclusive results on the exact relationship.

Fig. 12 also attests that the quasi-steady approximation is not a good estimate when the wake gets very close to the tail, and that in this case the wake rollup does indeed influence results, producing changes on both the location and the amplitude of the sudden lift variations. This is caused by extremely large induced velocities on the wake as it approaches the tail, due to the singularity in the Biot-Savart law. Note however, that except for the wake-tail encounters, the overall behavior has been predicted reasonably well without free-wake effects. In this analysis, a vortex-core has been defined for the regularization of the Biot-Savart law, and within the cutoff radius of the vortex segment the induced velocity equals zero. This is a rather violent approximation, but it provides a very robust numerical performance, which was deemed more critical here. If more details of the sudden changes of lift were needed, a rigorous convergence study would be required for temporal and spatial discretizations during the wake-tail impingement, and remeshing [16] or discarding wake connectivity $[17,22]$ should be considered. Viscous effects, however, are often important and are neglected by the model. In conclusion, the UVLM could be used to expose hazardous situations arising from this wake-tail direct interaction, but higher-fidelity analysis tools would be needed to further investigate these scenarios if they could compromise the design. 


\section{E. Wake interference on open-loop response}

Finally, the impact of the interference on the open-loop longitudinal dynamics of the free-flying aircraft will be assessed. The same HALE aircraft has been considered again, with $d_{p l}=1 \mathrm{~m}$, $d_{H T P}=2 \mathrm{~m}, \sigma_{1}=5$ and $\sigma_{2}=20$. It has been assumed that lift and structural weight remain locally balanced along the wing span, and hence gravity forces are only included in the payload. A sinusoidal elevator deflection is commanded around the trim configuration, and the time-domain response of the aircraft is monitored. This analysis is an open-loop version of that performed in Section V C: instead of prescribing the motions of the aircraft, a maneuver is commanded and the vehicle is free to follow the trajectory that will result from this input - note, however, that no particular path has been sought for.

First of all, the aircraft is trimmed for steady level longitudinal flight at a given velocity. The trim configuration is found using Newton's method with three inputs (angle of attack, $\alpha$, thrust per propeller, $T$, and elevator deflection, $\delta$ ) to zero the resultant longitudinal forces and moments. In this case $V_{\infty}=25 \mathrm{~m} / \mathrm{s}$ has been chosen, for which the corresponding trim values are $\alpha_{\text {trim }}=4.56$ $\mathrm{deg}, T_{\text {trim }}=2.42 \mathrm{~N}$, and $\delta_{\text {trim }}=9.85 \mathrm{deg}$. At these conditions, the tip deflection of the main wing is $z_{t i p}=1.06 \mathrm{~m}$. For the dynamic analysis, the elevator input will be given by

$$
\delta=\delta_{\text {trim }}+\delta^{*} \sin (\omega t)
$$

where the oscillation frequency is, as before, $\omega=5 \mathrm{rad} / \mathrm{s} . \delta^{*}= \pm \delta_{\text {trim }}$ have been chosen so that interference effects become visible, i.e., by making the wake pass close to the tail but avoiding direct collisions. Fig. 13(a) displays the flight trajectory during the first two periods of elevator perturbation. Snapshots every half-period are presented for both values of $\delta^{*}$, including the wake of the main wing, which represents the path followed by its root. On top is the case $\delta^{*}=\delta_{\text {trim }}$, which leads to a nose-down pitching at the beginning of the motion due to an increased force on the tail. The snapshots below are for $\delta^{*}=-\delta_{\text {trim }}$, where the aircraft pitches up first. More details are presented in Figs. 13(b) and 13(c), which show the lift coefficient of the tail and the resulting pitch rate of the aircraft, respectively, during these two cycles, with and without wake interference. For the case without interference, the influence of the wake shed by the main wing over the tail is switched off in SHARP - the influence over the shedding surface, i.e., the main wing, is accounted 


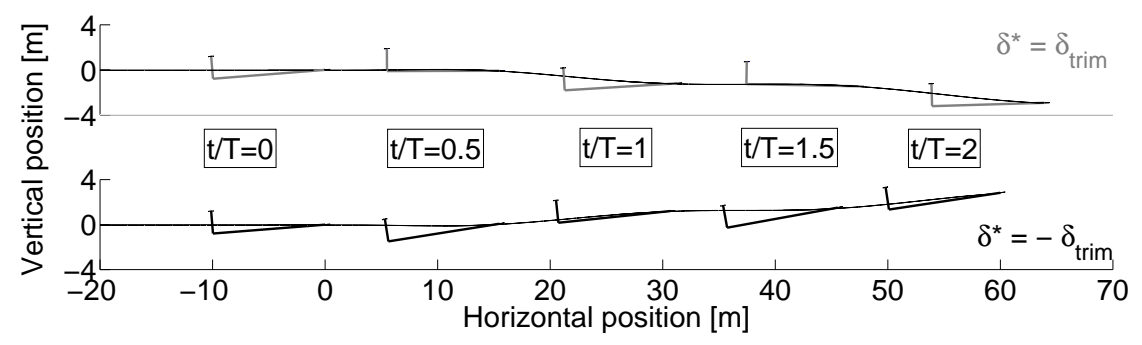

(a) Snapshots of the cross-section of the vehicle, including the wake-trajectory of the root of the main wing for $\delta^{*}=\delta_{\text {trim }}$ (top), and $\delta^{*}=-\delta_{\text {trim }}$ (bottom). Time at which snapshot was taken is included.

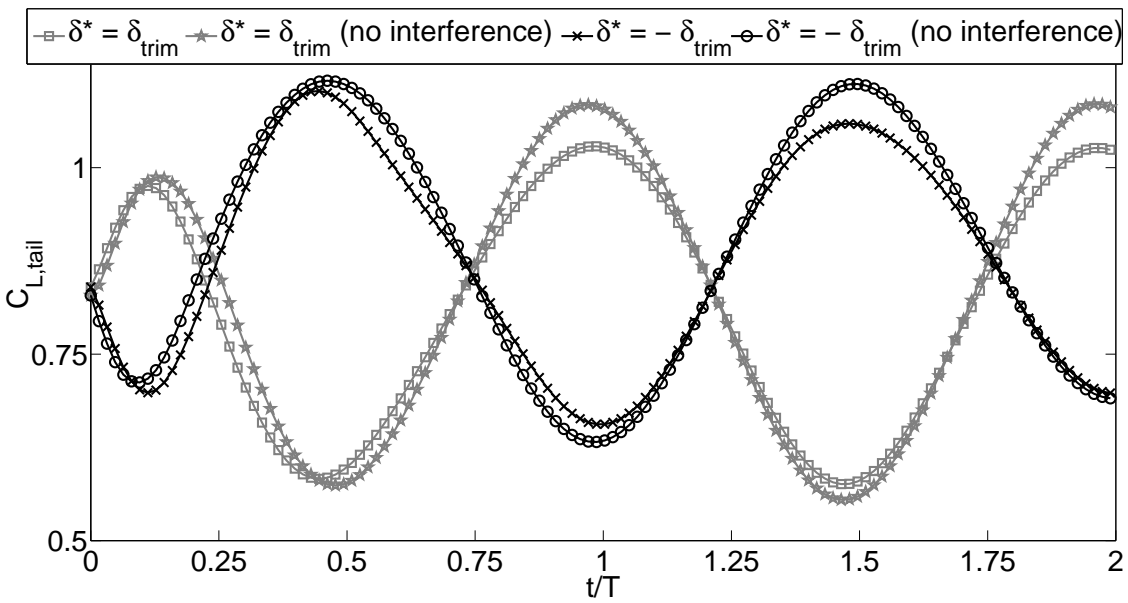

(b) Time evolution of tail lift coefficient for $\delta^{*}= \pm \delta_{\text {trim }}$ with and without interference.

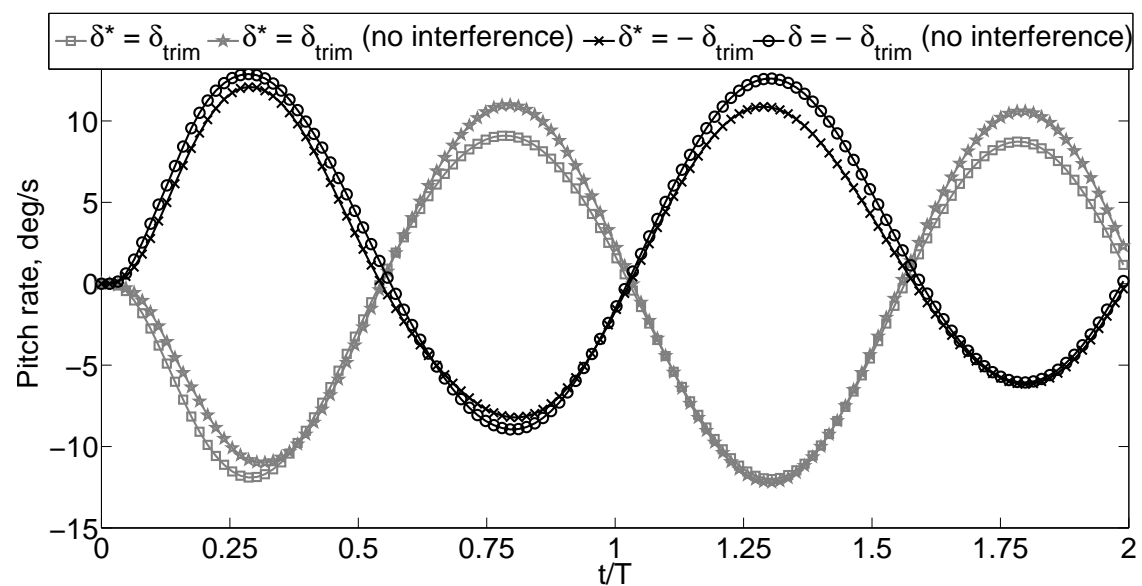

(c) Time evolution of pitch rate for $\delta^{*}= \pm \delta_{\text {trim }}$ with and without interference.

Fig. 13 Impact of wake proximity on the model HALE aircraft dynamics during two periods of elevator sinusoidal oscillation. $V_{\infty}=25 \mathrm{~m} / \mathrm{s}$.

for nonetheless.

The interference effect is apparent in these results, and shows the potential errors that can be 
introduced if the modeling neglects the dynamics of the wake. As the wake gets close enough to the tail, the induced downwash reduces the lift considerably and leads to a different pitch rate and trajectory; the error in pitch rate can reach up to $20 \%$ if interference is switched off. Note that for the parameters chosen the wake is always below the tail and there are no crossings. It was found that interference starts affecting significantly the results when the wake and the HTP are one chord length apart; otherwise, the results with and without interference are relatively close. In this case, the flexible aircraft was forced to follow a hard maneuver (indeed the maximum elevator deflection in the example exceeds $19 \mathrm{deg}$, which might lead in reality to flow separation), but similar results would be obtained with a flexible fuselage under vertical gust loads.

Finally, it should be remarked that no closed-loop control was attempted and, after the elevator input, a residual pitch rate persists that leads to a path that diverts from the trimmed state. This is also manifested by a smaller final forward velocity for $\delta^{*}=-\delta_{\text {trim }}$ that results in the aircraft covering a shorter distance.

\section{Conclusions}

This paper has presented a computational framework for the medium-fidelity modeling and simulation of low-speed flexible-aircraft dynamics at low-to-moderate frequencies. It couples 3-D unsteady vortex-lattice and geometrically-nonlinear composite-beam models to represent coupled aeroelastic and flight-dynamics responses. This approach captures the effects of large geometry changes, both in the structure and in the 3-D aerodynamics, but also coincides with more conventional linear models for small-amplitude dynamics. In addition, the model is able to capture a first (inviscid) approximation to the aircraft wake, and that has been used to investigate the interference between the wake of the wing and the aircraft tail for a HALE configuration. A loosely-coupled timemarching integration scheme has been implemented and exercised both for longitudinal prescribed rigid-body motions and open-loop response of the free-flying vehicle.

The effect of the wake of the main wing on the lift of the tail goes as the inverse of the relative distance between wake and tail. The numerical results in this work have shown situations in which this influence on the dynamics of the aircraft can be far from negligible. Also, a quasi-steady 
description of the wake has been often used in the literature to account for the induced downwash, but it has been observed here that the aerodynamic lags can play a relevant role on the dynamic response. Finally, the present coupled model can also provide some relevant information when direct collisions occur between wake and tail, even though the current modeling would be non-physical and allows the wake to pass through the tail. In these cases, the numerical results can only be seen as indicative of the actual physical event, but can provide valuable information during the design process.

The present model to study the dynamic response of flexible aircraft captures the large deformations of the lifting surfaces (as opposed to the doublet-lattice method), and includes the actual spanwise distribution of aerodynamic forces on the deformed wings, and the interactions with the free wake (as opposed to 2-D strip theory). This approach is expected to provide a suitable framework to incorporate these effects in the control synthesis, and to assess the performance of controllers designed with lower fidelity tools.

\section{Acknowledgments}

Joseba Murua gratefully acknowledges the financial support provided by the Department of Education, Universities and Research of the Basque Government. The authors would also like to thank the anonymous reviewers for their useful comments and for correctly indicating that the flutter results of the Goland wing in Ref. [34] are incorrect, and were overridden by those in Ref. [35]. These are the ones quoted in then final version of the paper.

\section{References}

[1] Drela, M., "Integrated Simulation Model for Preliminary Aerodynamic, Structural, and Control-Law Design of Aircraft," $40^{\text {th }}$ AIAA Structures, Structural Dynamics and Materials Conference, AIAA 991934, St. Louis, MO, USA, April 1999, pp. 1644-1656.

[2] Patil, M. J., Hodges, D. H., and Cesnik, C. E. S., "Nonlinear Aeroelastic Analysis of Complete Aircraft in Subsonic Flow," Journal of Aircraft, Vol. 37, No. 5, 2000, pp. 753-760. doi:10.2514/2.2685.

[3] Patil, M. J., Hodges, D. H., and Cesnik, C. E. S., "Nonlinear Aeroelasticity and Flight Dynamics of High-Altitude Long-Endurance Aircraft," Journal of Aircraft, Vol. 38, No. 1, 2001, pp. 88-94. doi:10.2514/2.2738. 
[4] Meirovitch, L. and Tuzcu, I., "Unified Theory for the Dynamics and Control of Maneuvering Flexible Aircraft," AIAA Journal, Vol. 42, No. 4, April 2004, pp. 714-727. doi:10.2514/1.1489.

[5] Garcia, J. A., "Numerical Investigation of Nonlinear Aeroelastic Effects on Flexible High-Aspect-Ratio Wings," Journal of Aircraft, Vol. 42, No. 4, July-August 2005, pp. 1025-1036. doi:10.2514/1.6544.

[6] Romeo, G., Frulla, G., Marzocca, P., and Tuzcu, I., "Non-Linear Aeroelastic Modeling and Experiments of Flexible Wings," $4^{\text {th }}$ AIAA/ASME/ASCE/AHS/ASC Structures, Structural Dynamics and Materials Conference, AIAA 2006-2186, Newport, RI, USA, June 2006.

[7] Shearer, C. M. and Cesnik, C. E. S., "Nonlinear Flight Dynamics of Very Flexible Aircraft," Journal of Aircraft, Vol. 44, No. 5, September-October 2007, pp. 1528-1545. doi:10.2514/1.27606.

[8] Wang, Z., Chen, P. C., Liu, D. D., and Mook, D. T., "Nonlinear-Aerodynamics/Nonlinear-Structure Interaction Methodology for a High-Altitude Long-Endurance Wing," Journal of Aircraft, Vol. 47, No. 2, 2010, pp. 556-566. doi:10.2514/1.45694.

[9] Su, W. and Cesnik, C. E. S., "Nonlinear Aeroelasticity of a Very Flexible Blended-Wing-Body Aircraft," Journal of Aircraft, Vol. 47, No. 5, 2010, pp. 1539-1553. doi:10.2514/1.47317.

[10] Palacios, R., Murua, J., and Cook, R., "Structural and Aerodynamic Models in the Nonlinear Flight Dynamics of Very Flexible Aircraft," AIAA Journal, Vol. 48, No. 11, November 2010, pp. 2648-2559. doi:10.2514/1.52446.

[11] Simo, J. C. and Vu-Quoc, L., "A Three-Dimensional Finite-Strain Rod Model. Part II: Computational Aspects," Computer Methods in Applied Mechanics and Engineering, Vol. 58, 1986, pp. 79-116. doi:10.1016/0045-7825(86)90079-4.

[12] Géradin, M. and Cardona, A., Flexible Multibody Dynamics: A Finite Element Approach, John Wiley \& Sons, Inc., 2001.

[13] Hodges, D. H., "Geometrically Exact, Intrinsic Theory for Dynamics of Curved and Twisted Anisotropic Beams," AIAA Journal, Vol. 41, No. 6, 2003, pp. 1131-1137. doi:10.2514/2.2054.

[14] Palacios, R., "Nonlinear Normal Modes in an Intrinsic Theory of Anisotropic Beams," Journal of Sound and Vibration, Vol. 330, No. 8, April 2011, pp. 1772-1792. doi:10.1016/j.jsv.2010.10.023.

[15] Noll, T. E., Ishmael, S. D., Henwood, B., Perez-Davis, M. E., Tiffany, G. C., Gaier, M., Brown, J. M., and Wierzbanowski, T., "Technical Findings, Lessons Learned, and Recommendations Resulting from the Helios Prototype Vehicle Mishap," NATO/RTO AVT-145 Workshop on Design Concepts, Processes and Criteria for UAV Structural Integrity, Florence, Italy, May 2007.

[16] Voutsinas, S. G., "Vortex Methods in Aeronautics: How to Make Things Work," International Journal of Computational Fluid Dynamics, Vol. 20, No. 1, 2006, pp. 3-18. doi:10.1080/10618560600566059. 
[17] Willis, D. J., Peraire, J., and White, J. K., "A Combined pFFT-Multipole Tree Code, Unsteady Panel Method with Vortex Particle Wakes," International Journal for Numerical Methods in Fluids, Vol. 53, 2007, pp. 1399-1422. doi:10.1002/fld.1240.

[18] Katz, J. and Plotkin, A., Low-Speed Aerodynamics, Cambridge Aerospace Series, Cambridge University Press, $2^{\text {nd }}$ ed., 2001.

[19] Elzebda, J. M., Mook, D. T., and Nayfeh, A. H., "Numerical Simulation of Steady and Unsteady, Vorticity-Dominated Aerodynamic Interference," Journal of Aircraft, Vol. 31, No. 5, 1994, pp. 10311036. doi: $10.2514 / 3.46606$.

[20] Wie, S. Y., Lee, S., and Lee, D. J., "Potential Panel and Time-Marching Free-Wake Coupling Analysis for Helicopter Rotor," Journal of Aircraft, Vol. 46, No. 3, May-June 2009, pp. 1030-1041. doi:10.2514/1.40001.

[21] Karkehabadi, R., "Aerodynamic Interference of a Large and a Small Aircraft," Journal of Aircraft, Vol. 41, No. 6, November-December 2004, pp. 1424-1429. doi:10.2514/1.4570.

[22] Cottet, G.-H. and Koumoutsakos, P. D., Vortex Methods: Theory and Practice, Cambridge University Press, 2000.

[23] Conlisk, A. T., "Modern Helicopter Aerodynamics," Annual Review of Fluid Mechanics, Vol. 29, 1997, pp. 515-567. doi:10.1146/annurev.fluid.29.1.515.

[24] Saban, D. and Whidborne, J. F., "Modeling of Wake Vortex Effects for Unmanned Air Vehicle Simulations," AIAA Modeling and Simulation Technologies Conference, AIAA 2009-5686, Chicago, IL, USA, August 2009.

[25] Mukherjee, R. and Gopalarathnam, A., "Post-Stall Prediction of Multiple-Lifting-Surface Configurations Using a Decambering Approach," Journal of Aircraft, Vol. 43, No. 3, 2006, pp. 660-668. doi:10.2514/1.15149.

[26] Døssing, M., Vortex Lattice Modelling of Winglets on Wind Turbine Blades, Master's thesis, Ris $\emptyset$ National Laboratory, Technical University of Denmark, Roskilde, Denmark, August 2007.

[27] Fritz, T. E. and Long, L. N., "Object-Oriented Unsteady Vortex Lattice Method for Flapping Flight," Journal of Aircraft, Vol. 41, No. 6, November-December 2004, pp. 1275-1290. doi:10.2514/1.7357.

[28] Leishman, J. G., Principles of Helicopter Aerodynamics, Cambridge Aerospace Series, Cambridge University Press, $2^{\text {nd }}$ ed., 2006.

[29] Dovgiř, S. and Shekhovtsov, A., "An Improved Vortex Lattice Method for Nonstationary Problems," Journal of Mathematical Sciences, Vol. 104, No. 6, May 2001, pp. 1615-1627. doi:10.1023/A:1011325112413. 
[30] Hodges, D. H., "A Mixed Variational Formulation Based on Exact Intrinsic Equations for Dynamics of Moving Beams," International Journal of Solids and Structures, Vol. 26, No. 11, 1990, pp. 1253-1273. doi:10.1016/0020-7683(90)90060-9.

[31] Palacios, R. and Cesnik, C. E. S., "Cross-Sectional Analysis of Non-Homogeneous Anisotropic Active Slender Structures," AIAA Journal, Vol. 43, No. 12, 2005, pp. 2624-2638. doi:10.2514/1.12451.

[32] Stevens, B. L. and Lewis, F. L., Aircraft Control and Simulation, John Wiley \& Sons, Inc., $2^{\text {nd }}$ ed., 2003.

[33] Farhart, C., Lesoinne, M., Stern, P. and Lantéri, S., "High performance solution of three-dimensional nonlinear aeroelastic problems via parallel partitioned algorithms: methodology and preliminary results," Advances in Engineering Software, Vol. 28, 1997, pp. 43-61. doi:10.1016/S0965-9978(96)00028-2.

[34] Goland, M., "The Flutter of a Uniform Cantilevered Wing," Journal of Applied Mechanics, Vol. 12, No. 4, 1945, pp. A197-A208.

[35] Goland, M. and Luke, Y. L, "The Flutter of a Uniform Cantilever Wing with Tip Weights," Journal of Applied Mechanics, Vol. 15, No. 1, 1948, pp. 13-20.

[36] Wang, Z., Chen, P. C., Liu, D. D., Mook, D. T., and Patil, M. J., "Time Domain Nonlinear Aeroelastic Analysis for HALE Wings," $4^{\text {th }}$ AIAA/ASME/ASCE/AHS/ASC Structures, Structural Dynamics, and Materials Conference, AIAA 2006-1640, Newport, RI, USA, June 2006.

[37] ZONA Technology, Inc., Scottsdale, AZ, USA, ZAERO Version 8.3, User's Manual, 2008.

[38] Smith, M. J., Patil, M. J., and Hodges, D. H., "CFD Based Analysis of Nonlinear Aeroelastic Behavior of High-Aspect Ratio Wings," 42 $2^{\text {nd }}$ AIAA/ASME/ASCE/AHS/ASC Structures, Structural Dynamics, and Materials Conference, AIAA 2001-1582, Seattle, WA, USA, April 2001.

[39] Smith, M. J., Schuster, D. M., Huttsell, L., and Buxton, B., "Development of an Euler/Navier-Stokes Aeroelastic Method for Three-Dimensional Vehicles with Multiple Flexible Surfaces," Structural Dynamics and Materials Meeting, AIAA 1996-1400, Salt Lake City, UT, USA, April 1996.

[40] Peters, D. A., Karunamoorthy, S., and Cao, W., "Finite State Induced Flow Models. Part I: TwoDimensional Thin Airfoil," Journal of Aircraft, Vol. 32, No. 2, 1995, pp. 313-322. doi:10.2514/3.46718.

[41] Yao, Z. X. and Liu, D. D., "Vortex Dynamics of Blade-Blade Interaction," AIAA Journal, Vol. 36, No. 4, 1998, pp. 497-504. doi:10.2514/2.406.

[42] Leishman, J. G., Bhagwat, M. J., and Bagai, A., "Free-Vortex Filament Methods for the Analysis of Helicopter Rotor Wakes," Journal of Aircraft, Vol. 39, No. 5, September-October 2002, pp. 759-775. doi:10.2514/2.3022.

[43] Tamura, A., Tsutahara, M., Kataoka, T., Aoyama, T., and Yang, C., "Numerical Simulation of Two- 
Dimensional Blade-Vortex Interactions Using Finite Difference Lattice Boltzmann Method," AIAA Journal, Vol. 46, No. 9, September 2008, pp. 2235-2247. doi:10.2514/1.30964.

[44] Horner, M. B., Saliveros, E., Kokkalis, A., and Galbraith, R. A. M., "Results From a Set of Low Speed Blade-Vortex Interaction Experiments," Experiment in Fluids, Vol. 14, No. 5, 1993, pp. 341-352. doi:10.1007/BF00189493. 